XII.

\title{
Die in den Jahren 1888 bis 1904 (bzw. 1911) in der Landes-Heil- und Pflegeanstalt für Geisteskranke in Hall in Tirol Verstorbenen und deren Todes- ursachen.
}

\author{
Von \\ Dr. Friedrich Plaseller,
}

Assistenzarzt.

Der vor kurzem erschienene, die Jahre 1905-1911 umfassende Jahresbericht unserer Anstalt brachte eine von mir über freundliche Anregung des Herrn Direktors, Sanitätsrats Dr. Josef Offer verfasste Abhandlung: Unsere Verstorbenen und deren Todesursachen. Ich sprach dort in den einleitenden Worten die Absicht aus, die Arbeit bis zum Jahre 1888, soweit eben die Sektionsprotokolle zurïckreichen, fortzusetzen, welches Vorhaben nun im Folgenden ausgeführt ist.

Es war, wie bemerkt sein möge, nicht immer ganz leicht, mir aus den oft spärlichen und inhaltsarmen Krankengeschichten das Bild der Psychosen zu vergegenwärtigen und eine den heutigen Anschauungen gerechte Diagnose zu stellen. Immerhin aber scheint es mir gelungen zu sein und ich gebe mich der Hoffnung hin, dass nicht alleuviele Fehlschlüsse unterlaufen sind.

Zu ganz besonderem Danke bin ich unserem gegenwärtigen Anstaltsleiter, dem Oberarzt Dr. J. M. Wassermann verpflichtet, der mir, wo Krankengeschichten ganz fehlten, ihr Inhalt karg. oder die Entscheidung sehr schwierig war, mit seinem umfangreichen Erinnerungsvermögen über einstige Patienten und seiner reichen klinischen Erfahrung stets hilfreich an die Hand ging.

So gelang es mir teils auch unter Zuhilfenahme der diagnostischen Bemerkungen im Sektionsprotokolle and dem Sektionsbefunde bei zwanzig fehlenden Krankengeschichten in 11 Fällen die vorgelegene Psychose festzustellen.

Bezüglich der Diagnosen wäre ich gern der für allgemeine Statistik vereinfachten Einteilung, wie sie Dr. Hans Roemer in Ilenau vor kurzem vorgeschlagen, gefolgt, wollte aber doch nicht von unserem Jahres- 
berichte abweichen. Dass ich mich dort nicht des uns vorgeschriebenen, längst veralteten statistischen Formulares bediente, ist wohl begreiflich.

Die Sektionen - es werden natürlich nur sezierte Fülle berücksichtigt - wurden in den ersten Jahren zumeist vom Universitätsprofessor für pathologische Anatomie in Innsbruck, Dr. G. A. Pommer, hernach vom Privatdozenten und späteren Universitätsprofessor Dr. Emanuel v. Hibler ausgeführt ${ }^{1}$ ).

Bezüglich der anderen Bemerkungen sei anf den Jahresbericht verwiesen. Auch hier möge es mir gestattet sein, interessante Krankheitsbilder mitzuteilen.

In den 17 Jahren nun, das ist von 1888 bis 1904, welche in erster Linie in Betracht gezogen werden, starben in der Anstalt 444 Kranke

Tabelle I.

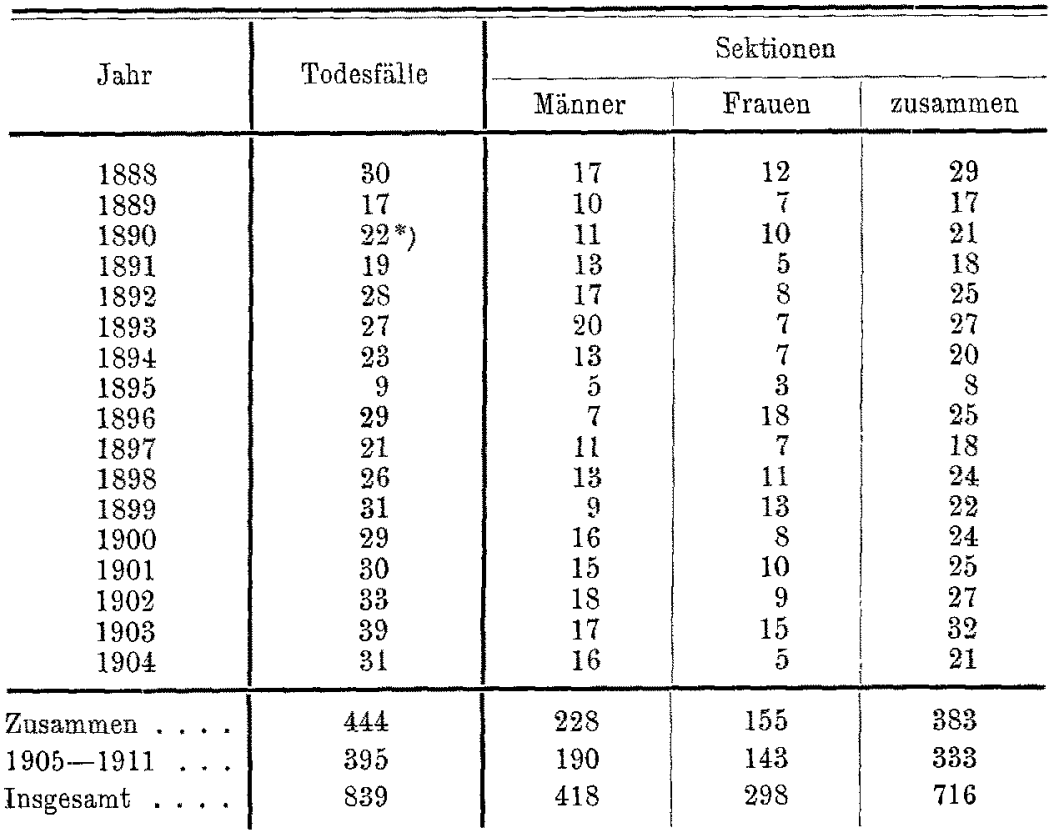

*) Diese Zahl wurde in den Sterbematrikeln der Stadtpfarre Hall erhoben. Bei der Seltenbeit, dass ein Patient anderer als katholiseher Kronfession in unserer Anstalt stirbt, wird die Zahl wohl als vollwertigg gelten können.

1) Mit Erlass des Tiroler Landesausschusses vom 24. 12. 1887 Z1. 16937 wurde Herr Professor Pommer damit betraut und ihm dabei eine jährliche Remuneration von $200 \mathrm{fl}$. verliehen, welche uber Beschluss des hohon Landtages in der Sitzung vom 9. 11. 1903 auf 600 Kronen und mit Landesaussohusserlass vom 3. 3. 1910 7. 715/VII auf 1000 Kronen erhöbt wurde. 
Die in den Jahren 1888 bis 1904 in Hall in Tirol Verstorbenen usw. 389

und von diesen wurden 383 seziert, 228 Männer und 155 Frauen. Im Zeitraum von 1888 bis 1911 verschieden insgesamt 839 Patienten und wurden 418 Männer und 298 Frauen obduziert, so dass also 716 Todesfälle zu unserer endgültigen Beobachtung zur Verfïgung stehen.

Aus der Tabelle I ist ersichtlich, dass im Jahre 1895 am wenigsten Kranke dem Tode erlagen, nur 9, von denen 5 Männer 'und 3 Frauen obduziert wurden. Am meisten Sterbefälle weist, das Jahr 1903 mit 39 auf, von denen an 32 die Leicheneröffnung vorgenommen wurde. Die übrigen Jahre bringen keine besonderen Schwankungen, nur das Jahr 1889 hat bloss 17 Verstorbene zu verzeichnen. In den Jahren 1889 und 1893 wurden alle Verstorbenen seziert, im Jahre 1900 hingegen am wenigsten, nämlich 21 von 31 . Durchschnittlich fallen also demnach 26 Todesfälle und 22 Sektionen auf das Jahr.

Eine wesentliche Steigerung der Todesfälle trat erst in den späteren Berichtsjahren mit der Erweiterung der Anstalt und der damit einhergehenden bedeutend vermehrten Krankenaufnahme ein.

Auf die einzelnen Monatsgruppen verteilen sich nun die Sektionen, wie Tabelle II zur Anschauung bringt.

Tabelle IL.

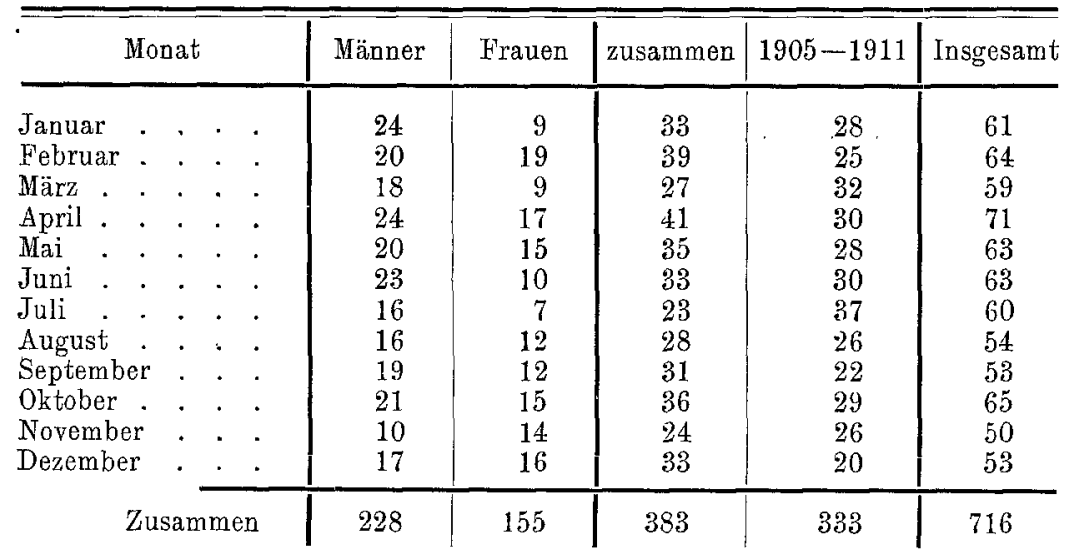

Daraus ist zu entnehmen, dass in den Julimonaten am wenigsten Frauen, nämlich 7, 1,82 pCt. von den Verstorbenen obduziert wurden, die meisten Männer hingegen in den Märzmonaten mit $24,6,29 \mathrm{pCt}$. Es kommen durchschnittlich auf einen einzelnen Monat zwei Sektionen.

Tabelle III bringt nun die Psychosen zur Ansicht, mit welchen die 383 obduzierten Fälle behaftet waren.

Es stellen also die Dementia praecox und die Dementia paralytica gleichviel Fälle, 105, 27,41 pCt., zusammen mehr als die Hälfte 
Tabelle III.

\begin{tabular}{|c|c|c|c|c|c|c|}
\hline \multirow{2}{*}{ Krankheitsform } & \multirow{2}{*}{ Männer } & \multirow{2}{*}{ Frauen } & \multirow{2}{*}{$\begin{array}{c}\mathrm{Zu}- \\
\text { sammen }\end{array}$} & \multicolumn{2}{|c|}{$1907-1911$} & \multirow{2}{*}{$\begin{array}{l}\text { Gesamt- } \\
\text { summe }\end{array}$} \\
\hline & & & & Männer & Frauen & \\
\hline Delirien . . . . . . . & 1 & 2 & 3 & - & 一 & 3 \\
\hline Alkoholismus . . . . . & 2 & 1 & 3 & 15 & 3 & 21 \\
\hline Dem praecox..... & 63 & 42 & 105 & 48 & 38 & $19 i$ \\
\hline Dem. paranoides .... & 9 & 8 & 17 & 4 & 4 & 25 \\
\hline Dem. paralytica .... & 85 & 20 & 105 & 46 & 19 & 170 \\
\hline Lues cerebri . . . . . & 1 & 一 & 1 & - & 1 & 2 \\
\hline Arteriosklerotische Ge- & & & & & & \\
\hline $\begin{array}{l}\text { hirnerkrankung . . } \\
\text { Dem apoplectica }\end{array}$ & $\overline{3}$ & 1 & $\begin{array}{l}1 \\
3\end{array}$ & 4 & 1 & 6 \\
\hline Traumatisches Irresein & - & - & - & 1 & 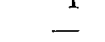 & $\begin{array}{l} \pm \\
1\end{array}$ \\
\hline Präsenile Demenz ... & - & 1 & 1 & 2 & -- & 3 \\
\hline Dem. senil. . . . . . & 19 & 16 & 35 & 30 & 26 & 91 \\
\hline Man.-depressiv. Irresein & 20 & 38 & 58 & 14 & 32 & 104 \\
\hline Paranoia ....... & 1 & 一 & 1 & 1 & 一 & 2 \\
\hline Epileptisches Irresein . & 11 & 6 & 17 & 19 & 7 & 43 \\
\hline Hysterie . . . . . . . & - & 2 & 2 & - & 1 & 3 \\
\hline Neurasthenie ..... & 1 & - & 1 & - & - & 1 \\
\hline Degeneratives Irresein. & 2 & - & 2 & - & - & 2 \\
\hline Imbecillitat. . . . . & 4 & 4. & 8 & 4 & 8 & 20 \\
\hline Idiotie . . . . . . . & 1 & 3 & 4 & 1 & - & 5 \\
\hline Tumor cerebri .... & - & 1 & 1 & 1 & - & 2 \\
\hline Irresein bei Pellagra & 一 & 1 & 1 & 一 & 1 & 2 \\
\hline Unbestimmte Diagnose & 5 & 9 & 14 & - & 1 & 15 \\
\hline Zusammen & 228 & 155 & 383 & 190 & 143 & 716 \\
\hline
\end{tabular}

aller. An ersterer beteiligen sich 63 Männer 27,63 pCt. $\left.(48,25,26 \mathrm{pCt} \text {. })^{1}\right)$ und 42 Frauen, 27,09 pCt. (38, 26,57 pCt.), bei letzterer 85 Männer, 37,28 pCt. (46, 24,21 pCt.) und 20 Frauen 12,9 pCt. (19, 13,28 pCt.). Es stimmen demnach die Ergebnisse der jetzigen Arbeit und die die Jahre 1905-1911 umfassenden annähernd überein bis auf die Anzahl der männlichen Paralytiker, die für letzteren Zeitabschnitt um 13,07 pCt. zurückgegangen sind. Bezüglich der Gesamtsumme sei erwähnt, dass auf die Dementia praecox $26,67 \mathrm{pCt}$, auf Dementia paralytica $23,88 \mathrm{pCt}$. entfallen.

Beim manisch-depressiven Irresein ist kein wesentlicher Unterschied gegenüber den früheren Zahlen, 58, 15,14 pCt. (46, 13,8 pCt.), 14,52 pCt. ${ }^{2}$ ). Auch hier sind die Frauen bedeutend stärker vertreten mit $38,24,51 \mathrm{pCt}$. (32, 22,37 pCt.) gegenüber den männlichen Kranken mit 20, 8,77 pCt. $(14,7,36$ pCt.).

1) Die Zahlen in Klammern bedeuten immer das Resultat der Jahre 1905 bis 1911.

2) Die fettgedruckten Zahlen beziehen sich auf die Jahre 1888-1911. 
Die Dementia senilis mit 35 Kranken, 9,13 pCt. bleibt um 7,69 pCt. gegenüber $56,16,82 \mathrm{pCt}$. in den letzten 7 Jahren zurück. Von der Gesamtsumme bilden die Alterskranken 12,7 pCt.

Aehnlich verhält sich die Epilepsie: 17, 4,43 pCt. gegen 26, 7,8 pCt. 6 pCt. und der Alkoholismus, 3, 0,78 pCt. (18, 5,4 pCt.) $\mathbf{2 , 9 3}$ pCt.

Die übrigen Psychosen weisen keine auffälligen Differenzen auf, nur die Dementia paranoides war von $8,2,4$ pCt. auf $17,4,43$ pCt. angestiegen; dies hat jedoch wenig Bedeutung, weil sie ja schliesslich doch eine Untergruppe der Dementia praecox vorstellt. Bezüglich der Paranoia muss ich erwähnen, dass der 54jährige Mann nach 21 tägigem Anstaltsaufenthalt starb, daher die Diagnose wohl nicht ganz einwandfrei erscheint. Sonst fand sich tatsächlich kein typischer Fall dieser Psychose.

Und nun mögen einige interessante Krankengeschichten folgen: Die drei Fälle von Delirium sollen entsprechend der Diagnosentabelle an erster Stelle stehen.

A. P., 31 Jahre altes Stubenmädchen wurde am 16. 4. 1903 wegen Pneumonie ohne nähere Angaben in die interne Klinik in Innsbruck gebracht, von der sie wegen ihres deliriösen Verhaltens noch am selben Nachmittag der psychiatrisch-neurologischen überwiesen wurde. Bei der Aufnahme an letzterer war sie anfänglich ganz ruhig, zeitlich und örtlich aber nicht orientiert. Körperlich war feststellbar: Gute Realtion der Pupillen auf Lichteinfall, rechtsseitige Pneumonie und über dem ganzen Herzen systolisches Geräusch.

17. 4. nachts wurde sie unruhig, wollte aufstehen, rief beständig nach ibrer Dienstfrau. Früh morgens zeigte sie ruhigeres Benehmen, kannte sich aber nicht aus. Am nächsten Tage setzte wieder die Unruhe ein, sie stand. auf, schrie, war nicht zu fixieren.

22. 4. Lungenbefund normal, jedoch grosse motorische Erregung, wälate sich sinnlos im Gitterbett herum, vollständig unzugänglich. Die Ueberbringung in unsere Anstalt konnte nur mit Zuhilfenahme des Spensers und dreier Begleitpersonen ermöglicht werden. Hier arbeitete sie den ersten Abend mit. Leibeskrätten, sich dem Wartepersonale zu entwinden, schrie in einem fort unzusammenhängende Worte, reagierte auf nichts; sie zerriss ihre Wäsche und das Bettzeug, schlug Purzelbäume, kroch auf dem Boden herum, bald auf dem Unterleib, bald rücklings, klopfte rhythmisch mit Händen und Füssen und wendete und drehte sich wie ein Wurm.

27. 4. Verhalten immer gleich. Sie springt gegen die Mauer, schlägt mịt dem Kopf wie mit einem Schlägel herum, steht Kopl, springt auf die Fensterbrüstung, stürzt sich mit dem Kopf voraus auf den Boden herunter. Aus der Nase rinnt Blut und Eiter, die Augenlider sind derart geschwollen, dass man die Bulbi nicht sehen kann. Dann jammert sie wieder über Kopfschmerzen, dabei redet sie ganz vernünftig. Ruft später wieder nach der Dienstfrau, in deren Umgebung sie sich wähnt. Sie meint verfolgt zu sein, 
man sperre sie ein, sie habe aber nie gestohlen, sie habe ihr Geld redlich verdient; gibt auch an, wo sie das Geld verwahrt hat. Nachdem sio sich beruhigt und im Bette geblieben, starb sie.

Sektionsbefund: Beiderseitige eitrige fibrinöse Leptomeningitis und Pachymeningitis interna. Hyperämie des Gehirns. Rechtsseitige lobuläre croupöse Pneumonie vorwiegend im Stadium der grauen Hepatisation mit konsekutiver fibrinöser Pleuritis, beginnende Pneumonie im linken Unterlappen, beginnende hämorrhagisehe linksseitige Pleuritis. Phlegmone des Zellgewebes der Galea.

Hier handelt es sich wohl um ein Kollapsdelirium.

M. Sch., 21 Jahre altes Dienstmädchen, wurde am 16.8. 02, nachdem sie im hiesigen Stadtspitale vom Arzte wegen Geisteskrankheit nicht aufgenommon worden war, der Anstalt iibergeben. Dem Begleitschreiben des behandelnden Arztes ist zu entnehnen, dass derselbe, Tags vorher zur Patientin gerufen, dieselbe nach stärkerem. Nasenbluten aufgeregt und irreredend getroffen habe. Dabei klaubte sie mit den Händen immer herum, sprach in einem fort selbstgemachte, bedeutungs- und sinnlose Worte. Die Kranke soll nach Angabe ihrer Angehörigen schon friiher nicht recht im Kopfe gewesen sein.

Bei der Aufnahme war sie sehr blass und blutarm, Temperatur 38,5. Die an sie gestellten Fragen wurden nur langsam beantwortet, sie schien sehwerhörig zu sein. Ihren Namen gab sie richtig an. Wo sind Sie angestellt? "Zu Hall in einem Ort." Bei wem? - "Bei Majer (richtig) oder wie es heisst. Ich weiss nicht, wie -". Die Kranke war zu weiteren Antworten nicht fähig. Das Bewusstsein unmebelte sich immer mehr und mehr und sie geriet schliesslich in einen förmlichen Sopor. Es entwickelte sich sehr schmerzhafte Nackensteife. Die Pupillen waren gleich weit, bewegten sich auf Lichteinfall, waren aber bald enger, bald weiter; Lidspalten gleich, rechter Mundwinkel hing etwas tiefer. Auf Nadelstiche sehr lebbafte Reaktion, Reflexe durchwegs gesteigert, vielfach mit lebhaftem Nachzittern. Babinski + . Ueber den Lungen bronchiales Atmen. Am 19. stellte sicl Cheyne-Stokesches Atmen ein, verbunden mit tonischer Starre der oberen Extremitäten.

20. 8. morgens $1 / 23$ Uhr exitus letalis unter Erscheinungen von Lungenodem in tiefer Somnolenz.

Bei der Sektion wurde festgestellt: Meningitis corebrospinalis tuberculosa. Gehirngewicht $1350 \mathrm{~g}$. Tuberkulose der Lungen, Nieren und Genitalien, miIiare Tuberkulose des Peritoneums.

Hier ist die Frage, welche Psychose durch Meningitis verdeckt wurde.

A. K., 44 Jahre alter Tagelöhner, wurde am 14. 6. 00 bewusstlos in der Höttinger Au aufgefunden und ins Innsbrucker Spital gebracht, von dort jedoch nach Hall überwiesen. Aus dem körperlichen Befund ist bemerkenswert: Pupillen eng, Reaktion nicht sicher prüfbar, Gesichtsinnervation ungemein schlaff, namentich die der rechten Wange. Die Zunge wich nach links ab. Rechter Mundwinkel tiefer. An der rechten Stirn eine Exkoriation. Kniesehnenreflex fast nicht auslösbar. 
Wie heissen Sie? - "Ist der Fackenlother auch da?" Haben Sie ein Weib? - "Liegst wohl du im Dreek da - ". Auf verschiedene Fragen erweisen sich seine Antworten so unartikuliert, dass sie nicht verständlich sind. Sucht mit den Händen immerfort im Betł herum. Gegen alle Veränderungen, die man mit ihm vornehmen will, wehrt und sträubt er sich ängstlich. Temperatur 38,9 .

15. 6. Fast fortgesetzt in komatösem bewusstiosen Zustand. Spricht oder murmelt unverständliche Worte, ist unrein, Schlueken erschwert, Nackenstarre. Am folgenden Tage wurde ex durch einen Wachmann agnosziert. Am 17. 6. gestorben.

Ergebnis bei der Leicheneröffnung: Meningitis eerebrospinalis purulenta bei Atrophie des Gehirns. Gehira $1250 \mathrm{~g}$ schwer.

Auch hier ist die Frage, welche Psychose ursprünglich vorlag ( $\mathrm{Pa}-$ ralyse?).

Mit Lues cerebri war ein 39 jähriger Kupfersehmied behaftet, bei dem schon längere Zeit beträchtliche Albuminurie bestand und der naeh 10 monatlicher Anstaltsbehandlung an 29. 2. 94 zum erstenmal von epileptiformen Krämpfen befallen wurde, welche in krampfhaften Augenstellungen nach aussen oben und klonischen Zuckungen der ganzen Körpermuskulatur bestanden. Die Anfälle - von der Dauer einiger Minuten - folgten sich in Zwischenräumen von cirea einer halben Stunde, bis er $3 / 41$ Uhr morgens des nächsten Tages denselben erlag.

Die Obduktion ergab: Pachy- und Leptomeningitis gummosa mit peripherer Enzephalomalacie des rechten Stirnlappens. Lues des Schädeldaches. Gummata in der rechten Lunge, Narben in der Leber. Chronische beiderseitige interstitielle Nephritis mit Uratinfarkten. Todesursache war die Nierenerkran. kung. Die Krämpfe dürften wahrscheinlich urämischer Natur gewesen sein.

Unter den Kranken mit postapoplektischer Psychose findet sich ein

35 Jahre alter, angeblich erblich nicht belasteter, lediger Baner. Derselbe fiel Ende Septernber 1900 plötzlich auf dem Felde um, war jedoch nicht bewusstlos, stand nach einigen Minuten wieder auf und begab sich nach Hause. Am selben Tage vermochte er noch ganz gut zu reden, am nächsten jedoch konnte er weder sprechen, noch aufstehen, war leicht verwirrt, erkannte aber seine Umgebung. Das Verchlucken auch flüssiger Nahrung war ihm beschwerlich, beide rechten Extremitäten waren vollständig gelähmt. In den nächsten 8 Tagen besserte sich der Zustand so bedeutend, dass or allein gehen konnte. Pat. verspürte sehon übers Jahr bei andauernder Arbeit Herzklopfen und Atemnot und litt viel an Kopfschmerzen. Potus wird geleugnet.

Am 28. 10.00 kam er in die psychiatrisch-neurologische Klinil in Innsbruck, wo der genane somatische und psychische Befund aufgenommen wurde, worauf einzugehen hier wohl nicht der Platz ist. Die Diagnose lautete: Embolie der linken A. foss. Sylvii (mittlere Aeste) und Insuffizienz der Aortaklappen. Im November desselben Jahres wurde er ziemlich gebessert entlassen.

Am 27.2. 01 brachte man ihn abermals dorthin, nachdem nach dem Verm lassen des Spitals noch weitere Besserung eingetreten war. Als aber seine 
Geliebte seinen Aufenthaltsort verliess, wurde er sehr aufgeregt, sprach ganz Unzusammenhängendes und Unzutreffendes. In der Klinik verbielt er sich zeitweise ruhig, dann aber auch sehr aufgeregt, wo er auf die Umgebung losschlug; die Kleider auszog und fortwährend meist ganz unverständliche Worte daherredete. Desgleichen schmierte er mit Kot. Die Aufnahrne seines psychischen Inventars war infolge der Dysarthrie der Sprache noch erschwerter. Es stellten sich schliesslich auch Gesichtshalluzinationen ein.

Am 15. 4. 01 wurde er an unsere Anstalt abgegeben, in der er am 21.8.01 starb. Eine hier geführte Krankengesehichte konnte ich nicht auffinden. Als Todesursache findet sich im Verwaltungsjournal Embolie angegeben.

Ergebnis der Leicheneröffnung: Embolie der rechten A. foss. Sylvii und Erweichung namentlich der Insel- und Operculumgebiete der linken Grosshirnhemisphäre. Exzentrisehe Herzhypertrophie bei Insuffizienz der Aortenklappen infolge grösstenteils vernarbter Endokarditis der rechten und linken Aortenklappen. Beiderseitiger Luugeninfarkt, Oedem der Lungen, Induration derselben, rechtsseitige Pleuritis.

Das im Lehrbuch der Psychiatrie von Dr. R. v. Krafft-Ebing, Band III, Stuttgart 1880 beschriebene Krankheitsbild einer Manischen soll hier kurz ergänzt werden. Dieselbe, eine

59 Jahre alte Sehustersgattin, kam am 17. 8.1877 (nicht 1879, wie es im Lehrbuch heisst) in unsere Anstalt. Es ist aus der Krankengeschichte zu ersehen, dass sich ihre "Tobsuchtsanfälle" fast mit genauer Regelmässigkeit alle 4 Wochen wiederholten und 2-3 Wochen anhielten, nur waren sie nicht immer von derselben Intensität, wahrscheinlich vielfach auch beeinflusst von Medikamenten, wie Bromnatrium, das bis zu $10 \mathrm{~g}$ pro die genommen wurde, Morphin- und Hyoseininjektionen. Der Beginn der Anfälle verriet sich zumeist durch kongestioniertes Aussehen der Kranken, gesteigerte Gesprächigkeit, durch Unruhe und Schlaflosigkeit und vielfach auch durch das Verlangen, entlassen zu werden. Sehr rasch entwickelte sich die Höhe der maniakalischen Erregung, in der sich Pat. Tag und Nacht in motorischer Unruhe befand, sang, schrie, die Aerzte in der unflätigsten Weise beschimpfte, aggressiv wurde, demolierte, die Kleider zerietzte, sich nackt auszog, unrein mit Urin war und mit Kot sehmierte. Das ideenflüchtige Gesehwätz zeigte viel sexuellen Inhalt. Später trat auch Sammeltrieb und die Manier, Gegenstände in den Abort zu werfen, hinzu. Ende der $80 \mathrm{er}$ Jahre verlängerten sich die anfallsfreien Zeiten bis gegen 2 Monate hin. In denselben arbeitete sie fleissig entweder auf der Abteilung oder auch beim Arzt in der Wohnung, sie litt jedoch viel an Kopfsehmerzen, Sehwindelzufällen und Obstipation. Nach dem Ablkingen der Aufregungen fïhlte sie sich immer am ganzen Leibe abgeschlagen. Sie wusste über alles Vorgefallene Auskunft zu geben, hielt aber an den dort erlebten Sinnestäuschungen fest.

Leider bricht die Krankengesehichte mit dem $5,6.1891$ ab. Am 13. 8.98 ereilte sie der Tod. Die Kranke war somit über 21 Jahre in der Irrenanstalt und daher am längsten unter allen Maniseh-Depressiven. 
Bei der Sektion fand sich: Braune Atrophie des Herzmuskels, fettige Durchwachsung desselben, Dilatation des Herzens, marantische Thrombose im rechten Ventrikel, völlige Embolie unł Thrombose der rechten und teilweise der linken Pulmonalarterie mit Infarktbildung in der rechten Lunge. Atrophie und Oedem des Gehirns mit geringem inneren und stärkerem äusseren Hydrocephalus, chronische Pachymeningitis ext. Gehirngewicht $1100 \mathrm{~g}$.

E. W., 30 Jahre alte, ledige Näherin, überstand in der Jugend - von erblicher Belastung nichts bekannt - sehr viele Kranleheiten: Rachitis, Scharlach, Morbilli, Blattern, Diphtherie, Arthritis rheumatica; im 6. Lebensjahre wurden ihr skrophulöse Drüsen herausgesehnitten, dreimal war sie „Iungenkranis".

Die Sehwester der Kranken gibt an, dass die Pat. wiederholt von Selbstmordabsichten gesprochen habe. Zu Hause habe sie nie Ruhe gefunden, hielt sich deshalb eine Zeitlang im Pflegehaus der barmherzigen Schwestern in Ried in Tirol auf. Fünfmal wurde sie bis zum Jahre 1900 an der psychiatrischen neurologisehen Klinik in Innsbruck wegen Hysterie behandelt. Im Juli desselben Jahres wurde sie dortselbst aufgenornmen, wobei sie angab, dass sie wegen zeitweise anftretenden Angstgefühlen mit leieht schrechbarem Wesen lomme. Die letzte Zeit vor der Aufnahme hätte sie fast jeden 5. Tag Anfälle gehabt, die ca. 20 Minuten gedauert hätten und denen eine längere Benommenheit gefolgt wäre; verletzt habe sie sich jedoch nie. Der Schlaf sei unregelmässig gewesen.

Körperlich war feststellbar: Rechte Pupille quer aval verzogen, Kniesehnenreflex erhöht, anch die übrigen Reflexe erböht, an der rechten Stirn und Wange Hypästhesie. Sie äussert das Gefühl, dass vom Magen eine Kugel gegen den Kehlkopf heraufsteige, weiter, dass von der Achselhöhle aus die oberen Extremitäten steif wïrden.

In der Klinik stellten sich wiederholt Anfälle ein, wobei das Bewusstsein stets erhalten blieb. Es zeigten sich Zuckungen an den Extremitäten und insbesondere Knirschen mit den Zähnen. Einmal verschluckte sie Sturckchen eines Rosenkranzes (Bete), dessen Messingteile grünspanig waren, um sich zu vergiften. Pat. war sehr leicht erregbar, jähzornig, beschimpfte das Warte. personal, wünschte sich den Tod und bat auch um ein Fläschehen Cyankali.

Am 28. 9. 00 warde sie unserer Anstalt überwiesen. Gleich am zweiten Tage erzählte sie, dass sie eine unglückliche Person sei, woran sie aber selbst Schuld habe, dadurch, dass sie immer ihrem Zorn nachgebe, die eigene Leidenschaft habe sie ins Irrenhaus gebracht und noch etwas - es helfe dagegen gar nichts -, nämlich die Onanie. Sie klagte über Schmerzen im Genitale, liess sich aber nicht untersuehen. Schliesslich aber am 17. 11., nachdem ihre Versuche vergeblich gewesen, wurde ibr vom Arzt ein schieferiges Holzstuck $15 \mathrm{~cm}$ lang, $1 \frac{1}{2} \mathrm{~cm}$ breit und $4,5 \mathrm{~cm}$ dick - aus der Vagina entfernt. Druekempfindlichkeit der Ovarialgegend. Gegenüber den anderen Kranken ist sie grob, beansprucht aber für sich selbst die grösste Aufmerksamkeit.

4. 12. Teilte dem Arzt mit, dass sie in Innsbruck auf einen Arzt ganz versessen gewesen sei, dass sie gemeint, sie müsse mit ihm geschlechtlich verkehren. Als ihr Antrag abgescblagen wurde, habe sie ihm versichert, sie wäre 
schon ïberglücklich beim Anblick seiner Genitalien. Sie trieb mitunter nach ihrer Angabe 12-15 mal Selbstbefriedigung am Tage. Oefters bekam sie opisthotonische Krämpfe, begleitet von Schüttelkrämpfen, wobei sie den herbeigerufenen Arzt beissen und kratzen wollte und mit der Pflegeschwester herumraufte. Andere Male warf sie der Pfiegerin die Esssehalen nach usw.

Am 28. 1.01 wurde sie gebessert entiassen, kam jedoch am 9.2. selbst wieder, weil sie sich durch ihre unglaublich oft ausgefïhrte Masturbation so schwach gefühlt habe, dass sie wähnte, am Ende ihres Lebens zu sein. Bie habe auch eine Freundin, eine geschiedene Frau, zu ähnlichen Zwecken besucht, aber nie dabei die Befriedigung gefunden wie bei der Selbstbefleckung. Sie verlangt vom Arzt Gift. Auf ihren Wunsch wurde ihr am 7.3. ein Ringpessar eingelegt, um die Selbstbefriedigung unmöglich zu machen.

17. 3. Sie gibt an, dass der Zweck erreicht sei und ist glücklich darüber. Am 24. 3. wurde das Pessar wegen Druckschmerzhaftigkeit entfernt, worauf sie innerhalb 4 Stunden 17-20 mal Selbstbefriedigung getrieben haben will, bat daher um ein anderes Pessar.

Im folgenden Frühjahr war sie sehr ruhig, beschäftigte sich vielfach in der Wohnung des Direktors. Sie trug einen Mutterring und behauptete, dass es ihr besser gebe, indem sie nur 2 mal im Monat sich selbst befriedigt habe.

Im Sommer wurde sie wieder aufgeregter, schimpfte auf Aerzte und Pflegepersonal; es konnte ihr nicht genug Aufmerksamkeit geschenkt werden. Zeitweise wieder gehäufte Onanie, worüber sie recht unglücklich war. Sie nahm sehr viel Medikamente, speziell Brom.

14. 4. 02. Verlangte das Wegnehmen der Klitoris wegen ihres furchtbaren Geschlechtstriebes.

16. 4. In Narkose Zerstörung der Klitoris mittels Thermokauter. Schon bald danach trieb sie wieder Selbstbefriedigung, bemerkte aber, dass dieselbe weniger „genussreich" und schwerer zu bewerkstelligen sei. Theatervorstcllungen, Unterhaltung, Bad, Zorn, unerfülte Wünsche, alles reize ihre Geschlechtslust. Nachdem sie sich wiederholt bereit erklärt, alles zur Heilung ihres Zustandes zu tun, wurde sie am 20. 10. 02 zur Kastration an die Frauenklinik nach Innsbruck geschickt, welehe dort auch vorgenommen wurde.

Aus der Klinik entlassen, begab sich Pat. in Privatpflege. Infolge eines schweren Anfalles im Januar 1903 wurde sie sehr traurig, äusserte Selbstmordideen und will erst von da ab manchmal masturbiert haben. Die Angehörigen überredeten sie in die Anstalt nach Hall zurückzukehren, welchem Rat sie auch am 17. 1.03 Folge leisteste. Sie war nun meist verstimmt, weil alles nichts helfe, wollte immer Gift, machte dem Arzt Vorwürfe über ihr Befinden, masturbierte aber selten. Es kamen auch Augenblicke, wo sie ihr Benehmen bereute und den Arzt um Verzeihung bat. Am 12. 4. brachte sie sich mit einem scharfen Instrument an verschiedenen Stellen beider Oberarme Sehnitte bei, um sich dann mit einem rostigen Nagel Blutvergiftung zu erzeugen, eilte aber selbst darauf zur Pflegerin, sich verbinden zu lassen. Bemerkt dazu, sie habe es nicht getan, um Gott zu beleidigen, sondern um sich von der bösen Leidenschaft zu befreien. Dann verlangte sie wieder vom Arzt digitale Vaginal- 
untersuchung und regte sich über die Ablehnung ihrer Bitte auf. Allerdings hatte sie mitunter Schmerzen im Bereich der Narben am Genitale. Anfälle hatte sie sehr selten.

Am 31. 8. kam sie zum Arzt aufs Zimmer zu einer Unterredung. Als der Arzt sich etwas abwandte, sprang sie auf die Fensterbrüstung und stürzte sich in den Garten hinunter (2 Stocknerke hoch). Sie wurde mit gestörtem Bewusstsein angetroffen, welches jedoch bald wiederkehrte. Gleich schimpfte sie, dass der Arzt ihr nichts gegen die Schmerzen gebe. Sie erkundigte sich, wie es mit ihr stehe und bat um Verzeihung, dass sie dies getan, es habe sie eigentlich schon gereut, wie sie den Kopf zum Fenster hinausstreckte. Es konnte ausser einigen kleineren Verletzungen nur ein Erguss ins linke Kniegelenk und eine höchstwahrscheinlich rechtsseitige Mandibularfraktur festgestellt werden.

Von da ab wurde Pat. immer kränklicher, klagte über Seitenstechen und hatte andauernd abendliche Temperaturerhöhung, nahm wenig Nahrung, magerte ab. Am Kiefer bildete sich eine kindskopforosse Geschwulst, die inzidiert wurde und woraus sich eine sezernierende Fistel bildete.

Mitte November wurde sie nachts sehr unruhig, verlor das Bewusstsein, zeigte Nackensteifigkeit, teilweise aufgehobene Reflexe, jedoch grosse Druckempfindlichkeit im geschwollenen Gelenk. Ueberhaupt jammert sie immer in gleichförmigen Tönen.

16. 11. Das Bewusstsein wurde immer trüber, die Kranke apathischer, die Sprache wurde lallend, undeutlich.

17. 11. Unter hochgradiger Dsspnoe, sehr starker Nackensteifigkeit und Erscheinungen von Lungenödem trat $1 / 210$ Uhr nachts der Tod ein.

Die Sektion ergab: Chronische, spärlich disseminierte Tuberkulose beider Lungen, Bildung einer kleinen Kaverne bzw. narbige Verdichtung an den Spitzen. Teilweises Oedem der Lungen, eitrige Bronchitis, Bronchiektasie. Atrophie und Dilatation des Herzens, Atrophie und Stauungserscheinungen in den grossen Körperdrüsen. Fraktur des linken äusseren Femurknochens, Vereiterung im Gebiete einer Frastur am rechten Unterkieferast und teilweise Nekrose des Kieferknochens. Chronisches Oedem der Meningen, Atrophie des Gehirns, Verknöcherungsherde an der linken Zentralwindung im Bereich der Mantelspalte. Leichter Hydrocephalus internus und granulöse Ependymitis.

Merkwürdig ist das Zusammentreffen von hypochondrischen Empfindungen mit jedenfalls wirklichen Schmerzen bei einem

43 jährigen Neurastheniker. Derselbe erscheint erblich nicht belastet. Im Alter von T-8 Jahren stürzte er von einem Heustadel herunter und seitdem war immer etwas Eigenartiges an ihm. Frühzeitig schon beschäftigte er sich mit Musik; die gewöhnliche Bauernarbeit gefiel ihm nicht, er wollte Lehrer werden; seinem Wunsch aber schenkte man kein Gehör. Während der aktiven Militärdienstzeit überstand er Typhus. Von 1880 an stand er auch als Musizer und Organist in verschiedenen Klöstern im Dienst, am längsten in Maria Plain bei Salzburg. Von da ab hielt er an keinem Posten recht lange mehr aus, nirgends passte es ihm, überall hatte er etwas auszusetzen, und war er an einem Ort, so fand er den früheren doch als den besseren und es reute ihn wieder, 
denselben verlassen zu haben. Zu Ostern 1896 wollte er Blutspicken gehabt haben, und schliesslich trat das Leiden im Anschluss an eine Erkäitung bei einem Versehgange vollends zutage: Die kousultierenden Aerzte sprachen von Magen-, Herz- und Leberleiden, bis schliesslich einer es als Nervosität erkannte und ihn nach Hall in die Anstalt schickte (30. 10. 96).

Bei der Aufnahme war Pat. sehr verzagt, jammerte, dass er in der Herzgegend immer eine Unruhe, einen Andrang habe, dass er nicht wisse, solle er liegen oder aufstehen. Wenn er etwas esse, sei es immer besser, Appetit sei. sehr gut. Bei der körperlichen Untersuchung wurde festgestellt: Lungenemphysem, der erste Spitzenton zu einem Geräusch verlängert, in der Magengrube grosse Schmerzhaftigkeit, Schleimhäute mit einem Stich ins Gelbliche, Obstipation. Pupillen prompt reagierend. Rechtes unteres Fazialisgebiet bodeutend schlaffer.

Der Schlaf erwies sich als sehr wechselnd, auch mit Hypnoticis, bald weniger, bald mehr. Manchmal will er bloss eine Stunde frühmorgens geschlafen haben. Immerhin schlief er vielmehr als er angab. Er jammerte über seine Schmerzen, kam bei Ablenkung doch immer wieder darauf zurück. Die Gesichtszüge waren oft schmerzhaft verzagt, der Blick jedoch leer, manchmal stellte sich momentan Schielstellung der Bulbi ein.

14. 11. Wurde durch einige Tage mit allgemeiner Massage behandelt, will bedeatend besser geschlafen haben.

17. 11. Wenn er sich vom Arzt beobachtet sieht, macht er allerlei Krümmüngen und Windungen, namentlich mit Vorliebe eine einseitig zusammengekauerte Stellung. Lenkt man ihn durch ein ihn interessierendes Gespräch ab, scheint er sich zu vergessen. Spontan spricht er nichts als von seinem Leiden.

23. 11. Wenn Magenwinde gehen, fürchte er halt immer, dass es ihm in der Herzgegend fehle. Schmerzen habe er immer.

30. 11. Zum ersten Male nachts Morphiuminjektion. Lubt von da an die Injektionen sehr. Manchmal auch ohne Schlafmittel fast keine Schmerzen gehabt. Eine Einspritzung mit Aq. destill. soll ihn sehr geschwächt haben, geschlafen habe er aber doch nicht. Hatte seine Gedanken stets bei seinen Schmerzen in den Füssen, im Magen, im Herzen. Probeweise zur Holzarbeit verwendet, geht es nicht recht, "es ziehe gerade soviel über die Schinken hinauf".

Mit Ende Dezember stellte sich Fieber ein, die Kräfte verfielen zusehends, der Schlaf wurde inmer geringer und am 30.12. 1896 starb er an Magenkrebs (siehe Tabelle $X$, Neoplasmen Nr. 12).

Bei der Gehirngeschwulst handelt es sich um eine

34 Jahre alte Bergmannsfrau, die 16 Tage nach der Aufnahme in die Anstalt verschied. Anamnestisch ist bekannt, dass sie seit einigen Monaten psychisch krank sei und an hysterischen Dämmerzuständen leide ...". Ueber den Anstaltsaufenthalt fand ich keine Aufzeichnungen, nur im Sektionsprotokoll ist die Diagnose: „Trresein mit Lähmung“ verzeichnet.

Die Obduktion erwies: Gliom des linken Stimhirns mit hochgradiger Usurierung des Schädels, Kompression des Nervus opticus. Bronchopneumonie bei bestehender Bronchitis und Bronchiektasie. 
Von den 14 Fällen mit unbestimmter Psychose waren bei 9 die Krankengeschichten nicht mehr auffindbar, bei den 5 übrigen keine Diagnose zu stellen. lch führe das vorhandene Material an:

1. A. N., 66 Jahre alter Strassenräumer, Potator, stand 1887 wegen Paralysis agitans an der internen Klinik in Innsbruck in Behandlung, aus der er wenig gebessert entlassen wurde. Dieser Krankheit schloss sich bald die Geistesstörung an. Pat. glaubte sich von Teufeln umringt, die ihm stark zusetzten. Gleichzeitig jammerte er, seine Seele sei infolge des schlechten Lebenswandels verloren. Oft schrie er stundenlang mit gellender Stimme, abwechselnd fluchend und betend. Nahrungsaufnahme und Schlaf gering. Am 14. 5. 1888 wurde er in unsere Anstalt abgegeben. Als einzige Bemerkung ist hier aufgezeichnet: Stets bettlägerig, schreit des Nachts, sehr hinfällig, Sprache gehemmt. Abends $2 \mathrm{~g}$ Chloralhydrat. Diagnose: Blödsinn. Exitus 4. 6. 1888.

Sektionsbefund: Atrophia cerebri mit Oedem der beiden Stirnlappen. Degeneratio grisea des Burdachschen Stranges der Medulla spinalis cervical. sup. Atrophia medull. spin. diffusa (Myel. chron.?). Gehirngewicht $1470 \mathrm{~g}$. Todesursache: Lungentuberkulose.

2. F. L., 49 Jahre alter lediger Bauer, erblich nicht belastet. Er war weder Trinker, noch Luetiker. Als Ursache der Erkrankung wird von dem behandelnden Arzte ein siebenjähriges Leberleiden und Ikterus angegeben. Die Geistesstörung trat schon öfters während der allgemeinen Wassersucht auf, verlor sich aber dann wieder mit Abschwellen derselben, bis sie das letzte Mal die Wassersucht überdauerte und nun 8 Monate anhielt. Pat. war leicht erregbar, heftig, boshaft, gewalttätig, tobend, zeitweise ganz geistesabwesend, manchmal von Verfolgungswahn eingenommen. Die letzten 14 Tage war er im Versorgungshause in Ried, Tirol. Pat. kam am 10.1. 1890 in ganz elendem Zustande in die Anstalt: Pulsschlag bei 160, in der Herzgegend ausgebreitete Dämpfung, Herztöne nicht wabrnehmbar. Die erste Nacht war er unruhig, zerriss seinem Nachbar den Rock. Die zweite Nacht scheint er, während der Pfleger kurze Zeit weg war, auf dem Boden herumgekrochen zu sein, wo er $63 / 4$ Uhr früh tot angetroffen wurde.

Im Gehirn fand sich bei der Sektion nichts Besonderes; als Todesursache wurde hämorrhagische serofibrinöse Perikarditis festgestellt. Die Leber zeigte Muskatnusszeichnung und vermehrtes Bindegewebe.

3. F. M., 59 Jahre alter Postunterbeamter, Potator wurde am 29. 7. 1892 in die psychiatrisch-neurologische Klinik in Innsbruck aufgenommen. Die körperliche Untersuchung ergab: Pupillen gleich weit, prompt auf Lichteinfall reagierend, mässiger Tremor der Zunge, Tremor der Hände, K. S. R. nicht auslösbar. Er antwortet auf alle Fragen mit nein. 31. 7. über den Lungenspitzen gedämpfter Schall, bronehiales Atmen und spärliche Rasselgeräuche.

9. 8. Pupillen reagieren sehr träge, B. D. R. gesteigert, eine in die Hand gegebene Nadel glaubt er noch zu besitzen, wenn man sie schon entfernt hat. Er gibt sie scheinbar von einer Hand in die andere und von da auf den Tisch. Taktile Reize mit der Nadel werden als "heiss" angegeben. Leitungsvermögen verlangsamt. Kein Fussklonus. Heben des Beines nur schwer unter ataktischen Bewegungen möglich. Ortssinn intakt. 
10. 8. Pat. etwas munterer, behauptet heute gehen zu können und will seit seinem Hiersein öfters im Garten gowesen sein. Der Versuch, aufzustehen, misslang wegen seiner grossen Aengstlichkeit. Seit vorigem Tage wässeriger Stuhl. Incontinentia urinae seit der Aufnahme, Sputa blutig. Diagnose: Dementia senilis, Alcoholismus chronicus, Tabes alcoholica.

13. 8. Aufnahme in die Anstalt in fast pulslosem Zustande. Aether bessert etwas die Herztätigkeit. Temperatur 38,5. Rasselgeräusche an der Lunge hörbar. Pat. murmelte wie im Halbschlaf etwas von Brixlegg, als man ihn nach der Heimat fragte, sonst ging er auf nichts ein. Am nächsten Tage abends $1 / 210$ Uhr gestorben, ohne dass aus ihm etwas herauszubekommen war.

Im Gehirn wurde bei der Sektion Verschmälerung der Rinde und ein Hirngewicht von $1300 \mathrm{~g}$ gefunden. Beginnende Pneumonie bei Myodegeneratio des. Herzens. Fettleber, sehr viel Fett im grossen Netz und in den Nierenkapseln, chronischer Magenkatarrb.

4. K. V., 27 Jahre alte Magd. Eine mütterliche Tante war trübsinnig, alle Geschwister etwas schwachsinnig, religiös ïberspannt. Ursache ihres „Verfolgungswahnes" soll schlechte Behandlung zu Hause gewesen sein. Weiteres. ist über ihren über 2 Jahre dauernden Anstaltsaufenthalt nicht zu finden.

Todesursache: Tuberkulose beider Lungen. Gehirn: Anämie und Oedem.

5. M. K., 70 Jahre alte Witwe, hatte einen Bruder, der am Ende seiner Studien geisteskrank geworden. Sie selbst soll zeitweise schwermütig gewesen sein. Zu Hause verweigert sie - seit einigen Wochen - oft tagelang die Nahrungsaufnahme und litt an Gesichts- und Gehörstäuschungen. Am 25. 7. 1898 wurde sie in die Anstalt aufgenommen. Hier hatte es immer Schwierigkeiten mit dem Essen, sie nahm die Nahrung erst, wenn man sagte, man gebe es sonst den Schweinen. War verzagt, müde, sprach wenig, nachts stand sie. manchmal auf, war aber ruhig, wenn auch schlaflos. Dabei war sie örtlich orientiert.

6. 8. Jammert meist, ist ganz verzagt. Das alles sei nichts für sie, sie gehöre nicht hierher. Das Bett sei zu gut, sie solle nichts essen, man solle nichts essen, man solle sich nicht zu ihr setzen. "Na, na, na, o Jesus Maria! Gehen Sie doch fort". - Starb denselben Tag noch.

Die Sektion wies nach: Atrophie des Gehirns, Sarkom der Tabula vitrea int. Myodegeneratio cordis.

Aus Tabelle IV wird die Verteilung der wichtigsten Arten derPsychosen auf die einzelnen Jahre ersichtlich gemacht. Es ergibt sich daraus, dass die vermehrte Anzahl der Sektionen nicht durch gleichmässige Beteiligung der Psychosen zustande gekommen ist, sondern dass einzelne Gruppen ganz bedeutend zugenommen haben, so ganz speziell der Alkoholismus, weiters die Dementia senilis, Epilepsie und Imbezillität.

Die Tabelle $\mathrm{V}$ gibt übersichtlich das Alter der Verstorbenen an. Die jüngste Patientin war eine 17 Jahre alte Kranke, von der die Krankengeschichte fehlt. Es wird später davon gesprochen werden, ebenso. 
Tabelle IV.

\begin{tabular}{|c|c|c|c|c|c|c|c|c|}
\hline Jahr & 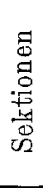 & 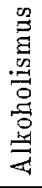 & 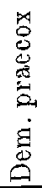 & 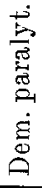 & 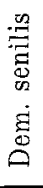 & 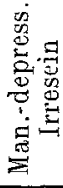 & 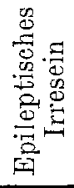 & 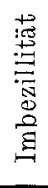 \\
\hline 1888 & 29 & - & 13 & 2 & 2 & 6 & 一 & - \\
\hline 1889 & 17 & - & 3 & 4 & 3 & 3 & 2 & - \\
\hline 1890 & 21 & - & 7 & 1 & 1 & 4 & 2 & - \\
\hline 1891 & 18 & - & 3 & 9 & 1 & 2 & - & - \\
\hline 1892 & 25 & - & 7 & 7 & 4 & 4 & 2 & - \\
\hline 1893 & 27 & - & 7 & 12 & - & 4 & 2 & - \\
\hline 1894 & 20 & - & 7 & 6 & 2 & 2 & - & 1 \\
\hline 1895 & 8 & - & 1 & 4. & - & 1 & - & 2 \\
\hline 1896 & 25 & - & 11 & 4 & - & 6 & - & 2 \\
\hline 1897 & 18 & $\ldots$ & 4 & 7 & 2 & 3 & - & - \\
\hline 1898 & 24 & 1 & 7 & 6 & 2 & 4 & 1 & 1 \\
\hline 1899 & 22 & $\ldots$ & 6 & 4 & 2 & 4 & 1 & - \\
\hline 1900 & 24 & - & 4 & 10 & 1 & 4 & 一 & - \\
\hline 1901 & 25 & 1 & 8 & 2 & 4 & 4 & 1 & - \\
\hline 1902 & 27 & - & 5 & 10 & 5 & 1. & 3 & - \\
\hline 1903 & 32 & 1 & 8 & 10 & 3 & 3 & 3 & - \\
\hline 1904 & 21 & - & 4 & 7 & 3 & 3 & - & 2 \\
\hline 1905 & 38 & 1 & 11 & 10 & 3 & 5 & 5 & 2 \\
\hline 1906 & 32 & 2 & 11 & 7 & 8 & 2 & - & 1 \\
\hline 1907 & 55 & 6 & 8 & 10 & 10 & 10 & 3 & 2 \\
\hline 1908 & 48 & 2 & 13 & 10 & 7 & 4 & 4 & 1 \\
\hline 1909 & 48 & 4 & 10 & 13 & 9 & 3 & 6 & 2 \\
\hline 1910 & 55 & 1 & 14 & 9 & 10 & 12 & 6 & 1 \\
\hline 1911 & 57 & 2 & 19 & 6 & 9 & 10 & 2 & 3 \\
\hline
\end{tabular}

kommen wir noch auf eine ebenfalls 17 Jahre alte Epileptikerin zurück. Eine dritte, ebenso alte manische Kranke erlag der Lungentuberkulose. Der jüngste unter den verstorbenen Männern war ein 20 Jahre alter Bauernsohn, der im epileptischen Dämmerzustande mit zahlreichen An'fällen an Lungengangrän starb. Die ältesten Anstaltspatienten waren zwei Frauen, eine die 82 jährige, oben beschriebene Manische und eine 83jährige, bei der im Anschluss an eine Herniotomie die rein demente Form ihrer senilen Demenz in heftige Erregung iuberging und die zwei Monate nach der Entwicklung letzterer einer Lungenentzïndung: erlag. Das Gehirngewicht war auf $1050 \mathrm{~g}$ gesunken. Der älteste Mann war ein 80 jähriger, ausserordentlich mürrischer, schimpfender, altersblöder Pfründner, bei dessen Obduktion ausser Pneumonie als Todesursache gefunden wurde: Enge des rechten Sinus transversus, ausserdem Arthritis deformans columnae vertebralis, vorzüglich des unteren rechten Brustanteiles.

Die Dementia praecox trifft man zwischen dem 21. und 70. Lebensjahr stets mehr weniger häufig vor. Bezüglich des Alters der ver- 
$\mathrm{Ta}$

\begin{tabular}{|c|c|c|c|c|c|c|c|c|c|c|c|c|}
\hline \multicolumn{3}{|c|}{ Alter } & $\begin{array}{l}\stackrel{g}{\Xi} \\
\stackrel{\Xi}{\Xi} \\
\stackrel{\Xi}{\Xi}\end{array}$ & 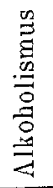 & 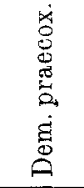 & 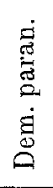 & 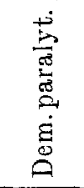 & 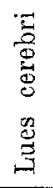 & 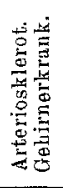 & 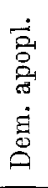 & 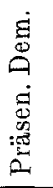 & 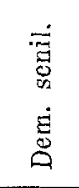 \\
\hline $\begin{array}{l}\text { Bis zu } \\
21-30 \\
31-40 \\
41-50 \\
51-60 \\
61-70 \\
71-80 \\
81-90\end{array}$ & $\begin{array}{c}20 \text { Jah } \\
\text { Jahre } \\
" \\
" \\
" \\
"\end{array}$ & $\begin{array}{l}\text { ren } \\
\cdots \\
\cdots \\
\cdots \\
\cdots \\
\cdots \\
\cdots\end{array}$ & $\begin{array}{l}- \\
0,1 \\
0,1 \\
1,0 \\
- \\
- \\
-\end{array}$ & $\begin{array}{l}- \\
\overline{1,0} \\
1,1 \\
- \\
- \\
-\end{array}$ & $\begin{array}{c}0,2 \\
19,4 \\
16,10 \\
10,17 \\
9,6 \\
8,2 \\
1,1 \\
-\end{array}$ & $\begin{array}{l}- \\
\overline{0,1} \\
3,2 \\
3,2 \\
1,3 \\
2,0 \\
-\end{array}$ & $\begin{array}{r}\overline{1,0} \\
28,5 \\
37,6 \\
13,8 \\
6,1 \\
- \\
\end{array}$ & $\begin{array}{l}- \\
1,0 \\
- \\
- \\
- \\
-\end{array}$ & $\begin{array}{l}- \\
- \\
\overline{-} \\
\overline{0,1} \\
-\end{array}$ & $\begin{array}{l}\overline{-} \\
1,0 \\
\overline{1,0} \\
1,0 \\
-\end{array}$ & $\begin{array}{l}- \\
- \\
\overline{0}-1 \\
- \\
-\end{array}$ & $\begin{array}{r}- \\
- \\
- \\
1,3 \\
7,6 \\
11,6 \\
0,1\end{array}$ \\
\hline Zusamy & nen. & & 1,2 & 2,1 & 63,42 & 9,8 & 85,20 & 1,0 & 0,1 & 3,0 & 0,1 & 19,16 \\
\hline
\end{tabular}

storbenen Paralytiker vergleiche man Tabelle Vl, aus der hervorgeht, dass die meisten im Alter zwischen 41-б̃0 Jahren starben, ein Ergebnis, das auch mit anderen derartigen Untersuchungen übereinstimmt. Die Dementia senilis kommt natürlich erst in den letzten Lebensdezennien zur Geltung, das manisch depressive Irresein wohl in allen Altersklassen, während die Epileptiker das Greisenalter nicht erreichen.

Ein grosser Teil der Kranken starb also im Alter zwischen 31-50 Jahren, $178,46,47$ pCt. (143, 42,94 pCt.), 44,83 pCt. Im neunten Dezennium finden sich noch zwei Kranke, 0,52 pCt. $(8,2,4 \mathrm{pCt}$.), $1,39 \mathrm{pCt}$, im achten $33,8,61 \mathrm{pCt} .(29,8,7 \mathrm{pCt}$.), $8,64 \mathrm{pCt}$. Bis zum 20. Jahre verschieden $5,1,3 \mathrm{pCt}$. $(61,8 \mathrm{pCt}$.), $\mathbf{1 , 5 5} \mathrm{pCt}$.

Tabelle VI.

\begin{tabular}{|c|c|c|c|c|c|c|}
\hline Alter & & $\begin{array}{c}1888 \text { bis } \\
1904\end{array}$ & $\%$ & $\begin{array}{c}1905 \mathrm{bis} \\
1911\end{array}$ & $\%$ & $\begin{array}{c}\text { Gesamt- } \\
\%\end{array}$ \\
\hline \multirow[t]{2}{*}{$\begin{array}{l}\text { ebensdezennium } \\
\text { do. } \\
\text { do. } \\
\text { do. } \\
\text { do. } \\
\text { do. }\end{array}$} & 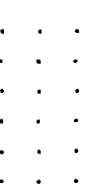 & $\begin{array}{r}-1 \\
33 \\
43 \\
21 \\
7\end{array}$ & $\begin{array}{r}-\overline{0,95} \\
31,42 \\
40,94 \\
20,00 \\
6,66\end{array}$ & $\begin{array}{r}1 \\
1 \\
22 \\
23 \\
14 \\
4\end{array}$ & $\begin{array}{r}1,53 \\
1,53 \\
33,84 \\
35,38 \\
21,53 \\
6,15\end{array}$ & $\begin{array}{r}0,58 \\
1,17 \\
32,35 \\
38,82 \\
20,58 \\
6,47\end{array}$ \\
\hline & & 105 & - & 65 & - & - \\
\hline
\end{tabular}

Den Anstaltsaufenthalt macht Tabelle VII anschaulich.

Am kürzesten verblieben in der Anstalt 2 Kranke mit unbestimmter Diagnose, die oben schon beschrieben wurden, mit drei Tagen, weiter ein Paralytiker, der am dritten Tage erstickte. Die Delirien gingen 
belle V.

\begin{tabular}{|c|c|c|c|c|c|c|c|c|c|c|c|c|c|}
\hline 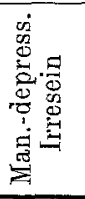 & 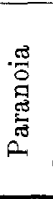 & 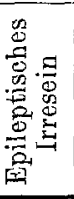 & 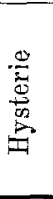 & 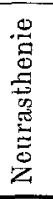 & 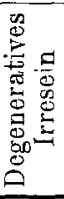 & 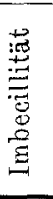 & $\begin{array}{l}\text { 量 } \\
\text { 总 }\end{array}$ & 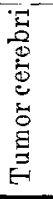 & 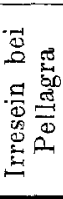 & 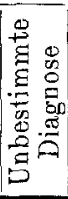 & 岁 & 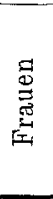 & 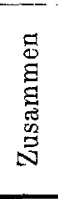 \\
\hline 0,1 & - & 1,1 & 一 & - & - & - & - & - & - & - & 1 & 4 & 5 \\
\hline & - & 3,0 & 0,1 & - & - & 0,2 & 1,2 & - & - & 0,1 & 24 & 11 & 35 \\
\hline 1,1 & - & 2,5 & 0,1 & - & 1,0 & 3,1 & 0,1 & 0,1 & - & 0,1 & 54 & 28 & 82 \\
\hline 5,5 & - & 3,0 & - & 1,0 & - & 0,1 & - & - & 0,1 & 1,1 & 62 & 34 & 96 \\
\hline 6,7 & 1,0 & 2,0 & - & - & - & 1,0 & - & - & - & 3,2 & 40 & 29 & 69 \\
\hline 7,15 & - & - & - & - & 1,0 & - & - & - & - & 1,1 & $3 ?$ & 29 & 61 \\
\hline 1,8 & - & 一 & - & - & - & - & - & - & - & 0,3 & 15 & 18 & 33 \\
\hline 0,1 & - & - & 一 & - & - & - & - & - & - & - & - & 2 & 2 \\
\hline 20,38 & 1,0 & 11,6 & 0,2 & 1,0 & 2,0 & 4,4 & 1,3 & 0,1 & 0,1 & 5,9 & 228 & 155 & 383 \\
\hline
\end{tabular}

nach 3-5 Tagen mit Tod ab. Ein maniakalischer, 44 Jahre alter Mann erlag am 4. Tage seines Anstaltsaufenthaltes einem Lungenödem, ein 35jähriger Wirt, Potator strenuus, mit akutem Delirium, wurde am 4. Tage der Anstaltsbehandlung um 5 Uhr früh ausserordentlich zyanotisch und bei bereits beginnender Totenstarre tot im Bette angetroffen. Die Sektion wies nach: Lungenödem.

16 Kranke, 4,18 pCt. (6, 1,8 pCt.), 3,07 pCt. wurden über 20 Jahre in der Anstalt verpflegt. Von diesen litten $12,75 \mathrm{pCt} .(5,83,33 \mathrm{pCt}$.), 77,27 pCt. an Dementia praecox. Am längsten hielt sich eine 66jährige Frau auf - 39 Jahre - deren Krankengeschichte mir leider unerreichbar war. Sie erlag der Dysenterie. 38 Jahre lang war ein 75jähriger Zimmermann, an Dementia praecox leidend, in der Anstalt, der an Typhus starb. Die mit Alkoholpsychosen Behafteten gingen innerhalb der ersten 3 Monate zugrunde. Ein grosser Teil der Praecoces erlebte einen Aufenthalt in der Anstalt von 2-10 Jahren, nämlich 35, 33,33 pCt. (36, 41,86 pCt.), $\mathbf{3 7 , 1 7}$ pCt. Eine ansehnliche Zahl der Paralytiker erreichte eine Aufenthaltsdauer von 6 Monaten bis zu 2 Jahren, 51, 48,57 pCt. (26,40 pCt.) , $\mathbf{4 5 , 2 9}$ pCt., also fast die Hälfte. Bei einem Aufenthalt von 2-5 Jahren starben verhältnismässig am meisten Kranke, $76, \quad 19,80$ pCt. $(60,18,01$ pCt. $), \quad \mathbf{1 8 , 9 9}$ pCt. Am wenigsten bei einem solchen von $15-20 \mathrm{Jahren}, 7,1,82 \mathrm{pCt} .(8,2,4 \mathrm{pCt}$ ), $\mathbf{2 , 0 9}$ pCt.

Ein 69 Jahre alter Mann mit postapoplektischem Blödsinn stand 19 Jahre in der Anstaltsbehandlung.

Sein Vater war Wirt, die Mutter litt an Schwermut, ein Bruder derselben und eine Tochter des letzteren waren geistesgestört. Ueberhaupt sollen Geisteskrankheiten in dieser Familie heimisch gewesen sein. Pat. kam schon früh 
T a -

\begin{tabular}{|c|c|c|c|c|c|c|c|c|c|c|}
\hline Anstaltsaufenthalt, & $\frac{0}{\stackrel{0}{\Xi}}$ & 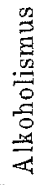 & 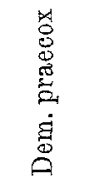 & 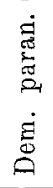 & 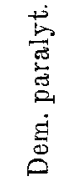 & 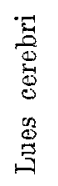 & 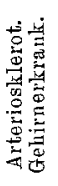 & 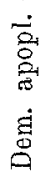 & 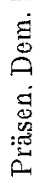 & $\begin{array}{l}\dot{\vec{\Xi}} \\
\dot{8} \\
\dot{0} \\
\dot{0}\end{array}$ \\
\hline 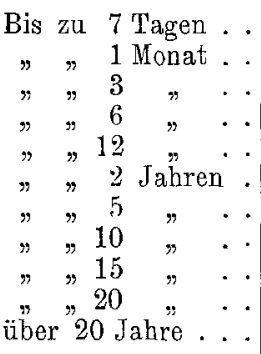 & $\begin{array}{l}1,2 \\
- \\
- \\
- \\
- \\
- \\
-\end{array}$ & $\begin{array}{l}1,0 \\
1,0 \\
0,1 \\
- \\
- \\
- \\
- \\
- \\
- \\
-\end{array}$ & $\begin{array}{c}\overline{1,1} \\
2,1 \\
5,1 \\
7,3 \\
4,4 \\
19,9 \\
14,13 \\
2,4 \\
0,3 \\
9,3\end{array}$ & $\begin{array}{l}- \\
\overline{1,0} \\
1,0 \\
\overline{1,2} \\
2,0 \\
2,2 \\
0,2 \\
0,2 \\
2,0\end{array}$ & $\begin{array}{r}3,0 \\
7,0 \\
8,3 \\
11,1 \\
20,5 \\
22,4 \\
11,6 \\
3,1 \\
- \\
-\end{array}$ & $\begin{array}{l}- \\
- \\
- \\
1,0 \\
- \\
- \\
- \\
-\end{array}$ & $\begin{array}{l}- \\
- \\
- \\
- \\
0,1 \\
- \\
- \\
-\end{array}$ & $\begin{array}{l}- \\
- \\
2,0 \\
- \\
- \\
- \\
\overline{1,0} \\
-\end{array}$ & $\begin{array}{l}- \\
- \\
\overline{-} \\
\overline{0,1} \\
\overline{-} \\
- \\
-\end{array}$ & $\begin{array}{l}2,0 \\
5,4 \\
3,2 \\
0,1 \\
4,2 \\
3,0 \\
2,5 \\
0,1 \\
\frac{-}{0,1} \\
-\end{array}$ \\
\hline Zusammen .... & 1,2 & 2,1 & 63,42 & 9,8 & 85,20 & 1,0 & 0,1 & 3,0 & 0,1 & 19,16 \\
\hline
\end{tabular}

hinters Trinken. Er verheiratete sich mit einer genusssüchtigen Frau und brachte schliesslich mit Wohlleben, Unordnung und Prozessieren sein Vermögen durch. Bei einem Bahnbau in Bayern musste er dann seinen Unterhalt verdienen. 1869 - 47 Jahre alt - traf ihn ein Schlaganfall; durch denselben verlor er teilweise das Gedächtnis und die Sprache. Er wurde von da ab ausserordentlich reizbar, zornjg, zankte sich mit den Leuten berum und bedrohte die Umgebung. Während der Pflege im väterlichen Hause besserte sich zwar etwas die Sprache, aber er wurde immer toller im Trinken, konnte fast keine Arbeit verrichten. Nachts schlief er wenig. Dazu kamen Wahnvorstellungen. Er glaubte sich in allerlei verkürzt, malte in ganz unverständlicher Weise seine vermeintlichen Forderungen auf und vertrat diese in ganz brutaler Weise. In einer solchen Aufregung wollte er sich am Grabe seines Vaters die Kehle durchschneiden, ein andermal sprang er in den hochgehenden Inn. Infolgedessen brachte man ihn am 16.12.72 in unsere Anstalt. Der rechte Arm fand sich in leichter Kontrakturstellung. Auch hier hatte er allerlei Grössenideen von seinen Geldern, war mitunter sehr aufgeregt, aggressiv, schimpfte schrecklich, demolierte Einrichtungsgegenstände, drängte hinaus, da er nicht in eine Trrenanstalt gehöre. Er machte wiederholt Fluchtversuche. Zeitweise aber arbeitete er auch etwas. Im Oktober 1878 bekam er die ersten epileptiformen Anfälle, die aber nur sehr selten sich wiederholten. Die letzten Jahre vor seinem Tode nahm das Gehörvermögen sehr ab, seine sprachlichen Aeusserungen wurden sehr gering und auch sehr schwer verständlich. Er sass, sein Pfeifchen schmauchend, glüctselig in einem Winkel herum. Am 20.1.91 starb Pat., nachdem er 11/2 Monate unter zunehmender Schwäche im Bette zugebracht und geringe Sehmerzhaftigkeit des Abdomens gezeigt hatte, an Karzinomatose des Peritoneums (s. Tabelle X, Nr.6). 
b e 11 e VIr.

\begin{tabular}{|c|c|c|c|c|c|c|c|c|c|c|c|c|c|}
\hline 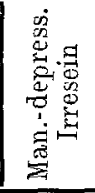 & 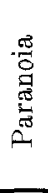 & 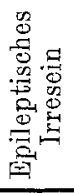 & 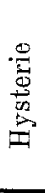 & 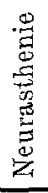 & 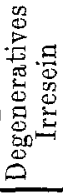 & 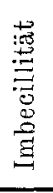 & & 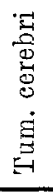 & 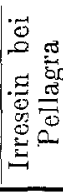 & 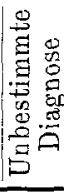 & 岕 & 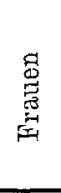 & 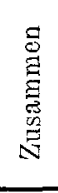 \\
\hline 3,1 & - & - & - & - & - & - & - & - & - & 2,0 & 12 & 3 & 15 \\
\hline 2,3 & 1,0 & 2,0 & - & - & - & - & - & 0,1 & - & 1,1 & 20 & 10 & 30 \\
\hline 4,2 & - & 1,0 & - & 1,0 & - & - & - & - & - & - & 20 & 9 & 29 \\
\hline 3,4 & - & 0,2 & - & - & - & 1,0 & - & - & - & 0,2 & 23 & 11 & 34 \\
\hline 1,8 & - & 3,0 & 0,1 & - & - & - & 0,1 & - & - & 0,1 & 36 & 21 & 57 \\
\hline 1,5 & - & 2,1 & - & - & 1,0 & 1,1 & 0,1 & - & 0,1 & 1,1 & 36 & 22 & 58 \\
\hline 4,7 & - & 1,2 & 0,1 & 一 & - & 1,3 & 1,0 & - & - & 0,2 & 41 & 35 & 76 \\
\hline 1,6 & - & 2,1 & 一 & 一 & 1,0 & 1,0 & 0,1 & - & - & 1,1 & 25 & 26 & 51 \\
\hline 1,1 & - & - & - & - & $\cdots$ & - & - & - & - & - & 3 & 7 & 10 \\
\hline 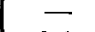 & - & - & - & - & - & - & - & - & - & - & 1 & 6 & 7 \\
\hline 0,1 & - & $\longrightarrow$ & - & 一 & - & - & - & 一 & - & 0,1 & 11 & 5 & 16 \\
\hline 20,38 & 1,0 & 11,6 & 0,2 & 1.0 & 2,0 & 4,4 & 1,3 & 0,1 & 0,1 & 5,9 & 228 & 155 & 383 \\
\hline
\end{tabular}

Die Sektion ergab folgenden Gehirnbefund: Basale Gefässe arteriosklerotisch; die erste rechte Temporalwindung, der grösste Teil der Inselwindung, der mittlere Teil der Zentralwindung zu braun pigmentierten, von Gefässen durchsetzten Defekten umgewandelt. Aehnliche Verhältnisse auf der linken Seite. Hirnwindungen atrophisch.

Im folgenden werden nun die Todesursachen besprochen. Tabelle VIII bringt sie anschaulich.

\section{Zentralnervensysitem.}

An Gehirnblutungen starben 4, 1,04 pCt., $\mathbf{0 , 9 7}$ pCt., 2 Männer, $0,86 \mathrm{pCt}$. und 2 Frauen $1,29 \mathrm{pCt}$.

Ein 71 Jahre alter senil dementer Mann erlag am vierten Tage nach dem apoplektischen Insult demselben. Bei der Sektion wurde nachgewiesen: Arteriosklerose der Gefässe, zirkumskripte Obliteration einer Kleinhirnarterie, apoplektische Herde in den linken zentralen Ganglien, zahlreiche kapillare Apoplexien in der Umgebung der grossen Ganglien und unter dem Ependym. Hämorrhagien der Basis des Kleinhirns.

Vier Stunden nach dem Schlaganfall verschied eine paranoide 50jährige Magd, deren Obduktion ergab: Hämorrhagien im rechten Linsenkern, in Thalamus opticus und in den Ventrikeln und intermeningeal.

An dritter Stelle reiht sich eine 52 jährige Kranke ein, die früher in heftiger manischer Erregung herumarbeitete, die letzten 2 Monate aber vollkommen ruhig war und ganz plötzlich im Saale tot zusammensank: Blutungen im Dache des vierten Ventrikels.

Im Alter von 78 Jahren fiel eine altersblöde Frau, gerade, während sie 
Tabelle VIII.

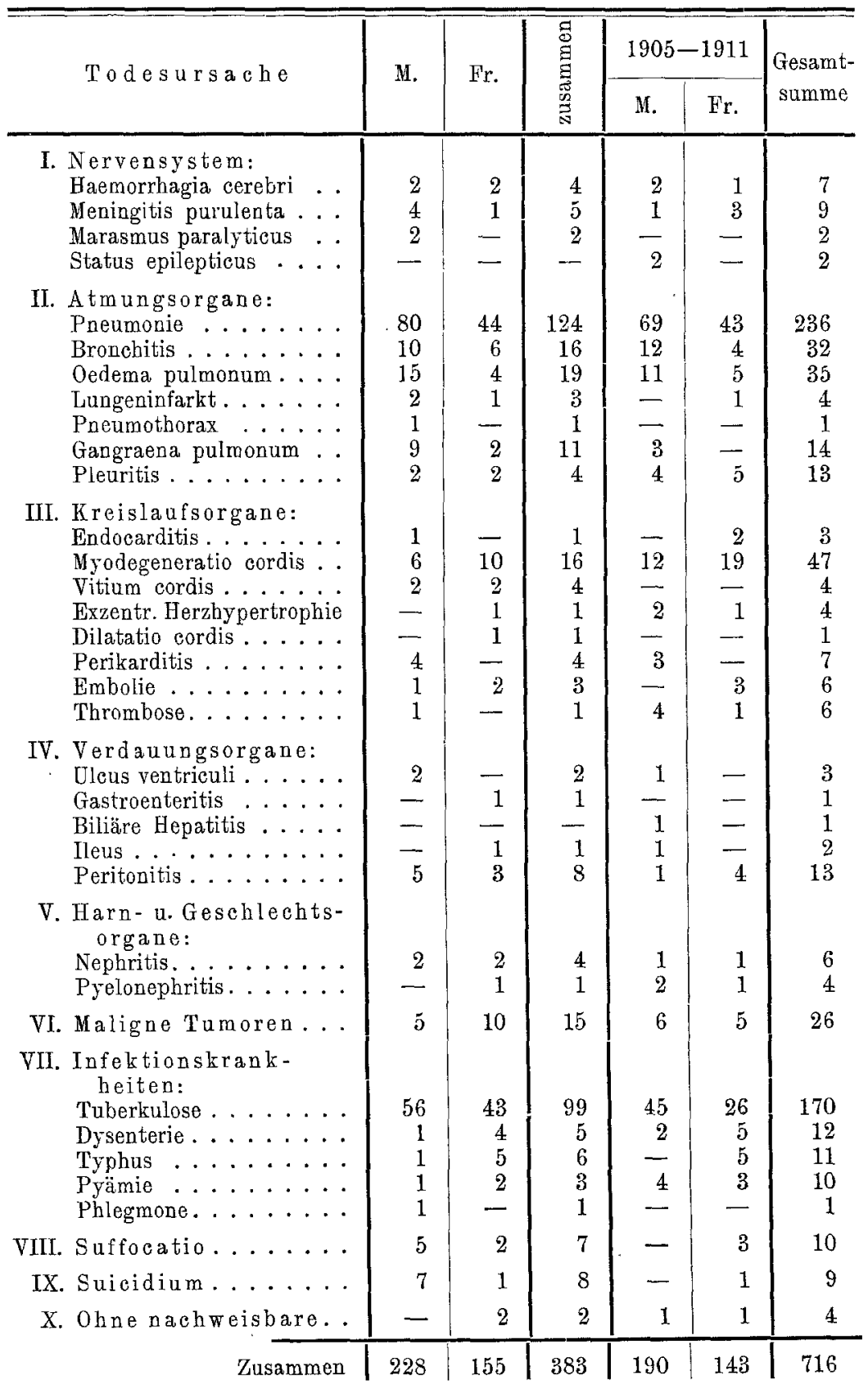


Die in den Jăhren 1888 bis 1904 in Hall in Tirol Verstorbenen usw. 407

sehrecklich schimpfte, bewusstlos zu Boden und starb 6 Stunden darauf: Blutherd im linken Schläfelappen und den Ventrikeln.

Meningitis führte in 5 Fällen zum Tode, 1,3 pCt. 2,04 pCt., von denen 2 schon beschrieben worden sind.

Bei einem 32jährigen Paralytiker entwickelte sich die septische, seröseitrige Hirnhautentzündung im Anschluss an bis in den linken Sitzbeinknorren vorgreifenden Dekubitus mit chronischem Abszess, zahlreiche, fast vernarbte dekubitale Veränderungen der Haut des Gesässes und Rückens. Eitrige Bronchitis.

An vierter Stelle kommt ein 61jähriger senil dementer Mann, bei dem am Schädel nichts Ursächliches gefunden werden konnte, wohl aber bestand lobuläre Pneumonie und serös-fibrinöse bämorrhagische Pleuritis. Als letzter schliesst sich an ein 46 Jahre alter Mann mit Dementia praecox behaftet, der eine serös-eitrige Otitis media neben rechtsseitiger Pneumonie hatte.

An Marasmus paralyticus starben 2. Es kounte bei denselben eine den Tod veranlassende Organerkrankung nicht festgestellt werden.

\section{A tmungsorgane.}

Die Lungenentzündung mit 124 Todesfällen $32,37 \mathrm{pCt}$., $\mathbf{3 2 , 7 6} \mathrm{pCt}$., erscheint wohl als die häufigste Todesart. Es sind allerdings die verschiedenen Arten der Pneumonien der Kürze halber zusammengezogen. Es entfallen davon 80 auf Männer, 36,08 pCt., und 44 auf dieFranen, $28,38 \mathrm{pCt}$.

Die Verteilung auf die einzelnen Psychosen ist durch Tabelle IX anschaulich gemacht.

Tabelle IX.

\begin{tabular}{|c|c|c|c|c|c|c|}
\hline Psgchose & Männer & Frauen & $\begin{array}{c}\mathrm{zu}^{-} \\
\text {sammen }\end{array}$ & $\begin{array}{c}1905 \text { bis } \\
1911\end{array}$ & $\begin{array}{l}\text { Gesamt- } \\
\text { summe }\end{array}$ & $\%$ \\
\hline 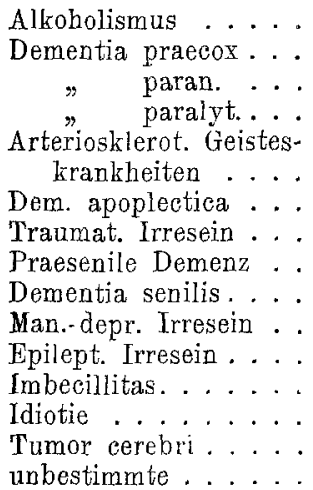 & $\begin{array}{r}\overline{8} \\
1 \\
42 \\
- \\
1 \\
-\overline{1} \\
\overline{11} \\
10 \\
3 \\
2 \\
1 \\
1\end{array}$ & $\begin{array}{r}-7 \\
3 \\
10 \\
1 \\
- \\
- \\
1 \\
7 \\
10 \\
1 \\
1 \\
1 \\
1 \\
2\end{array}$ & $\begin{array}{r}- \\
15 \\
4 \\
52 \\
1 \\
1 \\
1 \\
1 \\
18 \\
20 \\
4 \\
2 \\
2 \\
1 \\
3\end{array}$ & $\begin{array}{r}6 \\
13 \\
39 \\
4 \\
1 \\
1 \\
24 \\
12 \\
11 \\
-1 \\
-1\end{array}$ & $\begin{array}{r}5 \\
2 \\
1 \\
1 \\
42 \\
32 \\
15 \\
2 \\
2 \\
2 \\
3\end{array}$ & $\begin{array}{r}2,54 \\
11,86 \\
1,69 \\
38,55 \\
\\
2,11 \\
0,84 \\
0,42 \\
0,42 \\
17,79 \\
13,55 \\
6,35 \\
0,84 \\
0,84 \\
0,84 \\
1,26\end{array}$ \\
\hline Zusammen & 80 & 44 & 124 & 112 & 236 & - \\
\hline
\end{tabular}

Auf die Dementia paralytica trifft es von den an Lungenentzündung Verstorbenen 38,15 pCt., auf die senile Demenz 17,79 pCt., weiter ergibt 
ein Vergleich mit Tabelle III, dass fast die Hälfte aller Kranken mit obiger Diagnose an Poeumonie zugrunde gingen.

Die Bronchitis hat geringen Anteil, $16,4,17$ pCt., $\mathbf{4 , 4 6}$ pCt, 10 Manner, 4,37 pCt., 6 Franen, 3,87 pCt.

Dem Lungenödem erlagen $19,4,96 \mathrm{pCt} ., 4,88 \mathrm{pCt."} 15$ Mämer, 6.58 pCt.. 4 Frauen, 2,58 pCt.

3 Verstorbene, 0,78 pCt., $\mathbf{0 , 5 5}$ pCt., zeigten bei der Obduktion Lungeninfarkt, 2 Paralytiker und eine 68jährige manische Irre.

Gangrän der Lunge wurde $11 \mathrm{mal}$ angetroffen, $2,87 \mathrm{pCt.,} \mathbf{1 , 9 5} \mathrm{pCt}$, and zwar bei 9 Männern, 3,94 pCt., und bei 2 Frauen, 1,29 pCt. In vier Fällen wurde nichts ausser Gangrän festgestellt.

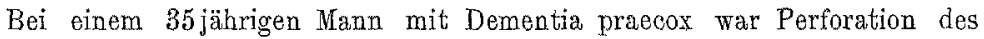
Oesophagus erfolgt. Ein 20jähriger Epileptiker zeigte bei der Sektion: Pyopneumothorax mit eitriger Infiltration der Bauchdechen bei Gangrän des Unterlappens der linken Lunge. Pneumomalacia dextra, Pericarditis und Mediastinitis purulenta. - Ein 47jähriger Paralytiker hatte einen handtellergrossen Dekubitus am Kreuzbein. - Ebenso Dekubitus am Ireuzbein und Trochanteren ein 34 Jahre alter paralytischer Mann. - Bei einer 49 jährigen schwermütigen Frau hatte sich die Gangrän aus käsiger Pneumonie entwickelt. Infolge Dekubitus am Kreuzbein trat bei einer 64jährigen Paralytikerin Gangrän der rechten Lunge auf, die wiederum infolge Durohbruehs Pleuritis erzengte. Ausserdem fanden sich bronchopneumonische Herde in beiden Unterlappen und gangränöse eitrige Bronchitis, Thrombose beider Kruralvenen. Der gangränöse Lnugenprozess führte bei einem 38jährigen Epileptiker zur Eröfnung eines grossen Gefässes und nachfolgender Verblutung; demarkierter embolischer Herd an der Spitze des rechten Schläfelappens. Zwei Blasenwürmer im Ependym bzw. unter demselben des rechten Ventrikels.

Pleuritis wurde bei 4 die Todesursache; 1,04 pCt., 1,81 pCt.. bei 2 Männern $(0,86 \mathrm{pCt}$ ) und bei 2 Frauen (1,29 pCt.).

Es kommen somit auf die Atmungsorgane 178 bzw. 335 Todesfälle, also nahezu die Hälfte.

\section{Kreislaulsorgane.}

An Endokarditis und zwar der Bi- und Trikuspidalklappen starb ein 45 jähriger manischer Kranker.

Der Herzmuskeldegeneration fielen 16 Kranke zum Opfer, 4,17 pCt., $\mathbf{6 , 5 6}$ pCt., 6 Männer, 2,63 pCt., und 10 Frauen, 6,45 pCt. Es erlagen also mehr Frauen als Mäner dieser Krankheit. Herzehler fuhrte 4 mal 'zum Tode, 1,04 pCt., 0,55 pCt., 2 Männex und 2 Franen. Ts handelte sich immer um Klappenfehler des linken Herzens. Bei einem 72 jährigen Weib wurde die exzentrische Herzhypertrophie zur causa mortis. Zu derselben gab aber hochgradige Arteriosklerose mit spezieller Beteiligung der Nieren Anlass. Ueber die Psychnse kann infolge fehlender Kranken- 
geschichte nichts gesagt werden, vielleicht handelt es sich um eine solche gleicher Natur. Gehirngewicht $1250 \mathrm{~g}$. - Ebenso selten war die Dilatatio cordis zum Tode führend, nämlich bei einer 62jährigen senil dementen Frau, wobei das ursächliche Moment hochgradige Atheromatose der Aorta bildete. - Herzbentelentziundungen verliefen viermal tödtlich; immer nur bei Männern und zwar dreimal als hämorrhagischserös-fibrinöse und einmal als hämorrhagisch-fibrinöse. - Von Embolie wurden 3 betroffen, ein Mann und zwei Frauen, 0,83 pCt. von den Gesamtverstorbenen. Zwei Fälle wurden schon oben beschrieben. - Eine 60 jährige manische Bäuerin erlitł am 21.11. 1890 eine Apoplexie, der sie am zweiten Tage darauf erlag: Embolie der A. carot. sin. und der A. fossae Sylvii mit nachfolgender Erweichung der entsprechenden Hirnpartien. - Thrombose wurde nur bei einem 41 jährigen Paralytiker und zwar der linken Pulmonalarterie beobachtet; von den Gesamtverstorbenen $0,83 \mathrm{pCt}$.

\section{Verdaungsorgane.}

Dem Magengeschwür erlagen 2 Männer, 0,41 pCt. von allen 716 Verstorbenen. Auch diesmal finden wir einen Fall von Ileus:

Bei einer manischen 47 jährigen Frau legte die Obduktion folgendes Bild klar: - die Krankengeschichte berichtet gar nichts über die letzte Aufenthaltszeit - Uterus unter Bildung zwoier Rezessus durch Stränge mit dem Peritoneum des hinteren Beckenraumes adhärent und in diesen waren Darmschlingen eingezwängt. Stauung in den Mesenterialgefässen und lokale Hämorrhagien in einzelnen Darmpartien; hochgradiger Meteorismus.

Peritonitis bildete bei 8 die Todesursache: $2,08 \mathrm{pCt} ., 1,81 \mathrm{pCt}$, bei 5 Männern $2,19 \mathrm{pCt}$. und bei 3 Franen.

1. 45 jähriger Paralytiler bekam die Peritonitis infolge Durehbruehs des Colon ascendens, worüber Näheres nicht zu eruieren ist.

2. Bei einer 48 Jahre alten Diurnistensgattin mit Dementia praecox wurde die Peritonitis haemorrhagica infolge einer obturierenden Thrombose der Aorta abdominalis bis zu den Nierenarterien reichend mit Gangrän der Gedärme und Teile des Magens hervorgerufen.

3. Bei einem 55jährigen paranoiden Mann war die Peritonitis beschränkt auf die Flexura sigmoidea vergesellschaftet mit chronisch vernarbender Tuberkulose der Lungenspitzen und obsoleter Pleuritis.

4. Bei einer 61 jährigen maniseben Frau entstand die grösstenteils organisierte und frische fibrinöse Peritonitis bei proliferierenden und zum Teil nekrotisehen, zum Teil vereiternden Kystomata der Ovarien.

5. Eine altersblöde 73jährige Frau bot bei der Leicheneröffnung folgendes Bild: Fibrinöse Peritonitis im kleinen Becken bei nekrotischer Proktitis, eitriger Periproktitis und Periaortitis und peripherer Cellulitis. Beginnende Perikarditis, erweichender Thrombus im Herzohr. Granulationsatrophie der Nieren und Hypertrophie des Herzens. 
Tabelle X.

\begin{tabular}{|c|c|c|c|c|c|c|}
\hline 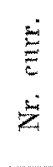 & 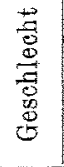 & $\begin{array}{c}\text { Psychiatrische } \\
\text { Diagnose }\end{array}$ & 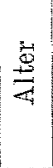 & 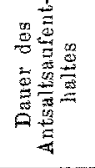 & $\begin{array}{l}\text { Primärer } \\
\text { Tumor }\end{array}$ & Bemerkung \\
\hline 1. & Fr. & $\begin{array}{c}\text { Dementia } \\
\text { praecox. }\end{array}$ & 65 & $\begin{aligned} \overline{5} \mathrm{~J} . \\
13 \mathrm{~T} .\end{aligned}$ & $\begin{array}{l}\text { Karzinom der } \\
\text { Portio vagi- } \\
\text { nalis uteri. }\end{array}$ & $\begin{array}{l}\text { Karzinomatöse Infiltration der Vagina } \\
\text { Cervix ateri und der Harnblase. Meta } \\
\text { stasen im Herz und Perikard und der } \\
\text { Halslympundrusen. }\end{array}$ \\
\hline 2. & Fr. & $\begin{array}{l}\text { Man-depress. } \\
\text { Irresein. }\end{array}$ & 63 & $\begin{array}{rr}5 & J \\
5 & \mathrm{M} \\
21 & \mathrm{~T}\end{array}$ & $\begin{array}{l}\text { Karzinom der } \\
\text { Cardia. }\end{array}$ & Profuse Blutung im Magen. \\
\hline 3. & Fr. & $\begin{array}{l}\text { Dementia } \\
\text { paranoides. }\end{array}$ & 44 & $\begin{array}{ll}5 & \mathrm{~J} \\
4 & \mathrm{M} \\
1 & \mathrm{~T}\end{array}$ & $\begin{array}{l}\text { Karzinom des } \\
\text { Pankreas. }\end{array}$ & $\begin{array}{l}\text { Karzinomatose der linken Nebenniere, de } \\
\text { Milz, des Magens und der linken Nieren } \\
\text { vene. Hibriaós-eitrige Peritonitis. }\end{array}$ \\
\hline 4. & Fr. & $\begin{array}{l}\text { Man-depress. } \\
\text { Irresein. }\end{array}$ & 74 & $\begin{array}{rr}2 & J \\
8 & \mathrm{H} . \\
13 \mathrm{~T}\end{array}$ & $\begin{array}{l}\text { Karzinom der } \\
\text { Ovarien. }\end{array}$ & $\begin{array}{l}\text { Karzinomatose des Pentoneums un } \\
\text { Zwerchfells mit serös-bämorrhagisch } \\
\text { fibrinösen Exsudaten im Bauchraum. }\end{array}$ \\
\hline 5. & M. & $\begin{array}{l}\text { Dementia } \\
\text { praecox. }\end{array}$ & 63 & $\begin{array}{l}4 \mathrm{~J} \\
4 \mathrm{M} \\
3 \mathrm{~T}\end{array}$ & $\begin{array}{l}\text { Medulläres } \\
\text { Karzinom des } \\
\text { Magens. }\end{array}$ & $\begin{array}{l}\text { Perforierend ins Colon transversum un } \\
\text { Jejunum. }\end{array}$ \\
\hline 6. & M. & $\begin{array}{c}\text { Demontia } \\
\text { apoplectica. }\end{array}$ & 69 & $\begin{array}{rr}18 & \mathrm{~J} \\
1 \mathrm{M} \\
5 \mathrm{~T}\end{array}$ & $\begin{array}{l}\text { Karzinom der } \\
\text { Gallenblase. }\end{array}$ & $\begin{array}{l}\text { Karzinomatose des Peritoneums, Meta } \\
\text { stasen in der Leber, Peritonitis chronic } \\
\text { et acuta. }\end{array}$ \\
\hline 7. & Fr. & $\begin{array}{l}\text { Man-depress. } \\
\text { Irresein. }\end{array}$ & 73 & $\begin{aligned} 9 \mathrm{~K} . \\
13 \mathrm{~T} .\end{aligned}$ & $\begin{array}{l}\text { Karzinom der } \\
\text { Mamma. }\end{array}$ & Metastasen in der Milz. \\
\hline 8. & Fr. & $\begin{array}{l}\text { Dementia } \\
\text { praeeox. }\end{array}$ & 54 & $\begin{array}{rl}25 & \mathrm{~J} \\
6 & \mathrm{H} . \\
3 & \mathrm{~T} .\end{array}$ & $\begin{array}{l}\text { Adenolrarzi- } \\
\text { nomatose der } \\
\text { Ovarien. }\end{array}$ & $\begin{array}{l}\text { Kystomata der Ovarien, Metastasen in de } \\
\text { Leber, fibrinos eitrige Peritonitis. }\end{array}$ \\
\hline 9. & M. & $\begin{array}{l}\text { Dementia } \\
\text { paranoides. }\end{array}$ & 58 & $\begin{array}{l}4 \mathrm{~J} \\
4 \mathrm{M}\end{array}$ & $\begin{array}{l}\text { Adenokarzi- } \\
\text { nom der Leber. }\end{array}$ & $\begin{array}{l}\text { Metastasen an der Wirbelsäule, Fom } \\
\text { pressionsmyelitis. }\end{array}$ \\
\hline 10. & Fr. & $\begin{array}{l}\text { Man.-depress. } \\
\text { Irresein }\end{array}$ & 58 & $\begin{array}{rl}1 & \mathrm{~J} \\
4 & \mathrm{M} \\
15 & \mathrm{~T}\end{array}$ & $\begin{array}{l}\text { Karzinom der } \\
\text { Mamma. }\end{array}$ & $\begin{array}{l}\text { Karzinomatose des Sternums, des } 3 \text {. Lenden } \\
\text { wirbels mit leichter Fixation der Duri } \\
\text { spinalis, Karzinomatose der Pleura un } \\
\text { Lober. }\end{array}$ \\
\hline 11. & M. & $\begin{array}{l}\text { Dementia. } \\
\text { praecox. }\end{array}$ & 67 & $\begin{aligned} 37 \mathrm{~J} \\
4 \mathrm{M}\end{aligned}$ & $\begin{array}{l}\text { Karzinom des } \\
\text { Rektums. }\end{array}$ & $\begin{array}{l}\text { Metastasen im Duetus choledochus un } \\
\text { in linken Hinterhauptbein. }\end{array}$ \\
\hline 12. & M. & Neurasthenie. & 43 & $2 \mathrm{M}$. & $\begin{array}{l}\text { Karzinom des } \\
\text { Magens. }\end{array}$ & - \\
\hline 13. & Fr. & $\begin{array}{l}\text { Man.-depress. } \\
\text { Irresein. }\end{array}$ & 76 & $\begin{array}{rr}5 & J \\
10 & \mathrm{M} . \\
24 & \mathrm{~T}\end{array}$ & $\begin{array}{l}\text { Karzinom des } \\
\text { Magens. }\end{array}$ & - \\
\hline 14. & Fr. & $\begin{array}{c}\text { Dementia } \\
\text { praecox. }\end{array}$ & 71 & $\begin{array}{rr}22 & \mathrm{~T} \\
1 & \mathrm{M} \\
20 & \mathrm{~T}\end{array}$ & $\begin{array}{l}\text { Karzinom des } \\
\text { Magens. }\end{array}$ & $\begin{array}{l}\text { Die Bauchaorta umbüllende Mestastase } \\
\text { der Leber. }\end{array}$ \\
\hline 15. & Fr. & $\begin{array}{l}\text { Man.-depress. } \\
\text { Irresein. }\end{array}$ & 51 & $11 \mathrm{~T}$. & $\begin{array}{l}\text { Karzinom der } \\
\text { Schilddrüse. }\end{array}$ & $\begin{array}{l}\text { Karzinomatose der Pleura, der rechter } \\
\text { Lunge und der Leber. }\end{array}$ \\
\hline
\end{tabular}


Die in den Jahren 1888 bis 1904 in Hall in Tirol Verstorbenen usw. 411

6. 44jähriger Paralytiker starb infolge Perforation seines chronischen Magengeschwüres und der sich anschliessenden fibrinös-eitrigen Bauchfellentzündung, während

7. ein anderer 64 jähriger Paralytiker nach Durchbruch eines tuberkulösen Geschwüres des Ileums der Krankheit erlag.

8. Fibrinös-seröse Peritonitis infolge Einklemmung des Ileums in seiner Mitte durch Bindegewebsstränge führte bei einem 79 jährigen Greis mit Dementia senilis zu dessen Ende: Pelveoperitonitis, Wurmfortsatz zu einem Bindegewebsstrang entlang dem Colon ascendens umgewandelt.

\section{Harn- und Geschlechtsorgane.}

An Nephritis starben 4, 2 Männer und 2 Frauen, $\mathbf{0 , 8 3}$ pCt., an Pyelonephritis ein Mann $\mathbf{0 , 5 5}$ pCt.

\section{Jaligne Tumoren.}

Bösartige Nenbildungen wurden 15 mal zur Todesursache, $3,91 \mathrm{pCt}$., $\mathbf{3 , 6 3}$ pCt. Die näheren Erläuterungen hierzu gibt Tabelle $X$. Es ist wohl kein Zusammenhang zwischen Psychosen und Neoplasmen zu finden.

VII. Infektionskrankheiten.

Hier sieht man die zweithäufigste Todesursache, die Tuberkulose, mit 99 Todesfällen, 25,84 pCt, $\mathbf{2 3 , 7 4}$ pCt. vertreten, 56 Männer, 24,56 pCt. und 43 Frauen, 27,74 pCt. Ob mehr die Männer oder die Frauen von Tuberkulose ergriffen wurden, scheint zu schwanken. Nachfolgende Tabelle XI führt uns die Beteiligung der Psychosen an dieser Krankheit vor Augen.

Daraus ergibt sich die grosse Anzahl von Tuberkulösen unter der Dementia praecox, ja mehr als die Hälfte aller Tuberkulösen fällt auf diese Psychose. Näheres zu der Anzahl der Verstorbenen der Tuberkulösen und der Dementia praecox gibt die Tabelle XII. Es nahm demnach trotz der Neubauten und der modernen Adaptierungen in der alten Anstalt die Tuberkulose nicht so wesentlich ab, und der Grund hierfür liegt eben in dem eigenartigen Verhalten der Praecoces.

Der Hauptsitz der Tuberkulose war bei 91 die Lunge, bei ó das Bauchfell, bei 2 die Meningen und schliesslich bei einem das Perikard. Andere Organe wurden ebenfalls tuberkulös angetroffen: 49 mal der Darmtrakt, 26 mal die Nieren, $19 \mathrm{mal}$ die Milz, 18 mal die Leber, 11 mal das Peritoneum, 8 mal die Wirbel, 6 mal die Meningen, 5 mal die Lungen, je 4 mal Rippen, Tuben und Blase, 3 mal die Prostata, je 2 mal das Grosshirn, der Kehlkopf, der Herzbeutel, der Magen und die Orarien, je einmal Zerebellum, Medulla spinalis, Tonsillen, Pharynx, Trachea, 
Tabelle XI.

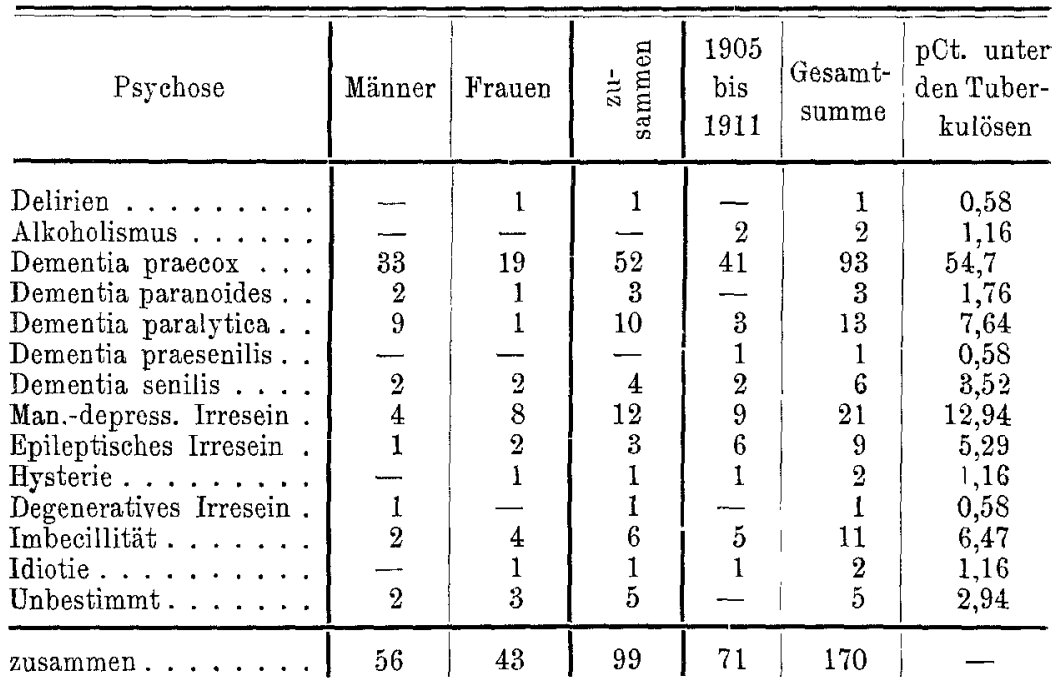

Tabelle XII.

\begin{tabular}{|c|c|c|c|c|c|c|}
\hline Jabr & Sektionen & $\begin{array}{l}\text { Tuber- } \\
\text { kulose }\end{array}$ & pCt. & $\begin{array}{c}\text { Dementia } \\
\text { praecox }\end{array}$ & $\begin{array}{c}\text { davon an } \\
\text { Tuberkulose } \\
\text { gestorben }\end{array}$ & $\begin{array}{l}\text { pCt. unter } \\
\text { den Tuber- } \\
\text { kulösen }\end{array}$ \\
\hline 1888 & 29 & 12 & 41,37 & 13 & 7 & 58,33 \\
\hline 1889 & 17 & 4 & 23,52 & 3 & 3 & 75,0 \\
\hline 1890 & 21 & 4 & 19,04 & 7 & 2 & 50,0 \\
\hline 1891 & 18 & 4 & 22,22 & 3 & 2 & 50,0 \\
\hline 1892 & 25 & 4 & 16,0 & 7 & 2 & 50,0 \\
\hline 1893 & 27 & 8 & 29,62 & 7 & 5 & 62,5 \\
\hline 1894 & 20 & 7 & 35,0 & 7 & 4 & 57,14 \\
\hline 1895 & 8 & 4 & 50,0 & 1 & 1 & 25,0 \\
\hline 1896 & 25 & 11 & 44,0 & 11 & 6 & 54,54 \\
\hline 1897 & 18 & 4 & 22,22 & 4 & 1 & 25,0 \\
\hline 1898 & 24 & 9 & 37,5 & 7 & 5 & 55,55 \\
\hline 1899 & 22 & 7 & 31,81 & 6 & 5 & 71,42 \\
\hline 1900 & 24 & 2 & 8,33 & 4 & 1 & 50,0 \\
\hline 1901 & 25 & 5 & 20,0 & 8 & 4 & 80,0 \\
\hline 1902 & 27 & 3 & 7,4 & 5 & 一 & - \\
\hline 1903 & 32 & 7 & 21,87 & 8 & 3 & 42,85 \\
\hline 1904 & 21 & 4 & 19,04 & 4 & 1 & 25,0 \\
\hline 1905 & 38 & 8 & 21,05 & 11 & 6 & 75,0 \\
\hline 1906 & 32 & 5 & 15,62 & 11 & 3 & 60,0 \\
\hline 1907 & 55 & 9 & 16,36 & 8 & 3 & 33,33 \\
\hline 1908 & 48 & 9 & 18,75 & 13 & 5 & 55,55 \\
\hline 1909 & 48 & 9 & 18,75 & 10 & 6 & 66,66 \\
\hline 1910 & 55 & 15 & 27,27 & 14 & 7 & 46,66 \\
\hline 1911 & 57 & 16 & 28,07 & 19 & 11 & 68,74 \\
\hline $\begin{array}{c}\text { qul- } \\
\text { sammen }\end{array}$ & 716 & 169 & - & 191 & 93 & 一 \\
\hline
\end{tabular}


Koronarvenen, Ureteren, Nebenhoden, Samenbläschen, Omentum, Uterus, Stirnknochen, Handwurzelknochen, Musculus rect. abdominis, Synovia eines Kniegelenkes. Zweimal fand sich ein Fungus eines Kniees, einmal ein solitärer Tuberkel der Gehirnbasis.

Ein 26jähriger, bereits ganz verblödeter schizophrener Bauernbursch starb am 23. 4. 1888 ganz plötzlich. Die Sektion wies nach: Luxation des Zahnfortsatzes des zweiten Halswírbels infolge Karies mit retropharyngealem Abszess. Tuberkulöse Basilarmeningitis. Chronische Lungentuberkulose. Residuen von Dysenterie des Kolons.

Ausgesprochene Tuberkulose - abgesehen von Drïsenschwellung und alten Narben - fand sich bei anderen Todesursachen noch $10 \mathrm{mal}$ in der Lunge, 3 mal im Darm, 2 mal am Knochen, 1 mal im Bauchfell und Perikard, zusammen 17 mal, so dass wir 116 Tuberkulöse, 30,28 pCt. unter den Verstorbenen gehabt haben, also fast ein Drittel. Cnter diesem Gesichtspunkte waren 203 von den gesamten Verstorbenen tuberkulös, 28,35 pCt.

Die Dysenterie holte sich 5 Kranke zum Opfer, 1 Mann und vier Frauen, auf verschiedene Jahre verteilt, so dass von einer Epidemie nicht gesprochen werden kann $(\mathbf{1}, \mathbf{6 7}$ pCt.). Mehrmals wurden dysenterische Darmveränderungen als Nebenbefund angetroffen. Der Typhus ging 6 mal tödtlich aus, bei 5 Frauen und 1 Mann 1,56 pCt., 1,53 pCt.; und zwar 5mal in den Jahren 1901-1903 und bis auf eine Hysterische immer bei Praecoces.

An Pyämie starben drei: 1 Mann und 2 Frauen, 1,39 pCt.

Bei einer 69 jährigen depressiven Frau ging sie aus von einer diffusen Gangrän des Unterschenkels und eitriger rechtsseitiger Gonitis. Es hatte sich weiter entwickelt: Thrombose der Arteria poplitea sinistra, bämorrhagische Peritonitis, Milztumor, Herzatrophie.

Die Sektion eines 59jährigen Paralytikers ergab: Septikopyämie. Fibrinöseitrige beiderseitige Pleuritis bei multiplen Abszessen in der Muskulatur der vorderen. Thoraxwand und Interkostalraum. Eitrige Bronchitis, lobuläre fibrinöse und kroupöse Pneumonie, Prostataabszess. Eitrige ulzerierte Proktitis. Vereinzelte Nierenabszesse, hämorrhagische eitrige Arthritis im rechlen Hüftgelenk bei Splitterfraktur des Femurhalses dieser Seite. Geringgradige Myodegeneratio. Die Verletzung hatte sich Pat. dadurch zugezogen, dass er am 25. 2. 1899 über die Lehne einer Bank steigen wollte, es aber nicht fertig brachte und rittlings hängen blieb: Der Tod trat am 12.6. ein.

Eine 44 jährige Paralytikerin hatte einen jauchigen Abszess im retroproktalen Zellgewebe und im Anschluss daran Verjauchung eines Stückes der hinteren Wand des Rektums. Ausserdem linksseitige akute Nephritis. Lobuläre frische und teils hämorrhagische pneumonische Herde in den hinteren Teilen der Lunge. Katarrhalische Geschwüre im Dickdarm. Thrombose der queren und S-förmigen Blutleiter der Schädelkapsel mit puriformer Erweichung. 
Die PhJegmone betraf einen 32 jährigen Epileptiker: Phlegmonöse Verdickung und Infiltration der Weichteile des Halses, besonders des subsutanen Gewebes mit Vergrösserung der Tonsillen, Oedem des Zellgewebes im Rachen mit Oedem der aryepiglottischen Falten, Pblegmone des Halses. Frische boginnende hypostatische Pneumonie.

\section{Suffocatio.}

Durch Ersticken gingen 7 Kranke zugrunde, 5 Männer und 2 Frauen, $1,82 \mathrm{pCt} ., 1,39 \mathrm{pCt}$.

1. 72 jährige schwerkranke, schwermütige Pat., verhielt sich viderspenstig gegen das Einschöpfen des Essens. Am 20. 2. 89 verfällt sie raseh nach Darreichung der Nahrung und starb bald darauf. Die Leichenöffnung ergab: Tod durch Aspiration von Speiseteilen in die Bronchien, Pleuritis exsudativa, haemorrhagica, Pericarditis haemorrhagica, Pneumonie, Oedema pulmonum, Herzdegeneration.

2. Während mehrerer hintereinander folgender paralytischer Anfälle aspirierte ein 36jähriger Kaminfeger Speiseteile aus dem Magen und erstickte.

3. Am 28. 10. 94 fand ein 38jähriger Paralytiler den Erstickungstod, indem er sich den Mund voll trockenen Gugelhupf stopfte. Der herbeigerufene Arzt kam schon zu spät.

4. Ein 42jähriger paralytischer Tagelöhner wurde am 23. 1. 97 zum drittenmal in die Anstalt aufgenommen, nachdem or im heimatiichen Spital wegen gesteigerter Erregung nicht mehr behalten werden konnte. Er schimpfte und fluchte in ganz schrecklicher Weise und musste wegen seiner Unruhe isoliert gehalten werden. Er würgte alles Essen furchtbar hastig hinein, sehien überhaupt kein Sättigungsgefühl zu haben. Am $2 S$ 1. erhielt er seine Knödel zu Mittag verabfolgt, und als der Pfleger nach etwa 10 Minuten die Schale abholen wollte, lag Pat. ohne Lebenszeichen auf dem Boden. Der Arzt konnte nur mehr Erstickung durch den eingeschoppten linödel feststellen.

5 . Ein 32 jähriger Schlosser, behaftet mit Paralyse, war die letzten Tage infolge Widerstrebens gegen jede Nahrungsaufnahme mit der Sonde gefiittert worden. Am 21, 6. 02 hatte er selbst genïgend gegessen, am 22. und 23. wurde ihm löffelweise eingeschöpft, er presste aber mit Gewalt jeden Tropfen. herauf, so dass mit der Ernährung ausgesetzt werden musste. Wasser und alles wies er mit entrüsteten Gebärden ron sich. Starb dann plötzlich. Bei der Sektion wurde angetroffen: Suffocatio bei Aspiration gallig gefärbten, flüssigen Mageninhaltes und chronische und akute Gastritis.

6. Eine 17jahrige Arbeiterstochter, Epileptikerin, hatte während ihres 3 monatigen Anstaltsaufenthaltes 17 beobachtete Anfälle; sonst gebärdete sie sich aufgeregt, schimptend, teilweise war sie auch verwirrt. Am 9.8. 02 wurde sie um 1/27 Uhr früh, nachdem man sie noch un 4 Uhr gehört zu haben glaubte, tot aufgefunden. Sie hatte wahrscheinlich einen Anfall und ist in demselben erstickt. Pat. lag mit dem Gesicht nach unten, dasselbe tief ins Kissen hineingedrückt. Beim Umkehren der Leiche sah man dic Totenflecken schon ausgebildet am Hals und an den Wangen, nicht aber um den Mund, an 
der Stirn und den ulnaren Teilen der Hände. Obduktionsergebnis: Ausgebildetes Oedem der Lunge und Hyperämie derselben, wie auch der Trachea und der Bronchien, des Rachens und Kehlkopfes, geringer Hydrothorax, Hydroperikard und Aszites. Tueichte akute Dilatation des Herzens, Enge der Aorta und Koronararterien, persistente Thymus, Struma substernale. Passive Hyperämie in den Drüsen der Bauchhöhle. Ueberall dunkles flüssiges Blut. Suffocatio. Chronisches Oedem der Meningen, Hyperämie der Hirnrinde und der Ganglien. Angeborene Kleinheit der letzteren. Adhäsive Pachymeningitis.

7. Ein 61jähriger Paralytiker erstickte durch Verlegung des aditus ad laryngem durch den ganzen Rachenraum erfüllende FiJeischmassen.

Daraus ist wahrzunehmen, dass bei den Paralytikern mit ihrer Unbeholfenheit die Todesursache sechsmal Erstickung war und nur einmal ein epileptischer Anfall bei einer Epileptikerin, im Verein mit Hypoplasie des Gefässsystems.

\section{Suicidium.}

Selbstmord begingen innerhalb der Anstalt 8 Patienten, 2,08 pCt., 7 Männer und 1 Frau, 1,25 pCt. Seit Eröffnung der Neubauten kam ein solcher nur einmal vor.

1. F. M., 27 jähriger Student phil., war mütterlicher- und väterlicherseits schwer belastet und schon vom 29. 10. 79 bis 10.7. 81 wegen Dementia praecox in Behandlung gestanden und als gebessert entlassen worden. Die Wiedereinbringung am 25. 7. 88 reranlasste ein Sprung des Pat. aus einem zweiten Stockwerk, wobei er sich eine Verletzung des Schultergelenkes zuzog. Er war die letzte Zeit in seinem Benehmen sehr wechselnd. Bald nahm er ganz sonderbare Stellungen ein, bald machte er sich Selbstverletzungen, zog sich nackt aus, war unrein mit Urin, später wieder verrichtete er Schreibarbeiten. Gemütlich zeigte er ein Gemisch von Glückseligkeit und höchstem Unglück, dann wieder verhielt er sich mutazistisch. - Am 25. 12. 88 erhängte er sich während des Friihgottesdienstes mit dem ledernen Hosenriemen an der Türschnalle des Abortes. Wiederbelebungsversuche waren erfolglos. Bei der Sektion wurde festgestellt: Die Gefässe der Pia waren strotzend mit Blut erfüllt, Plexus mit dunkelfüssigem Blut erfültt. Entsprechend der Strangulationsfurehe die Trachealschleimhaut suffundiert. Intima dor Halskarotis an einer Stelle abgelöst. Tuberkulose der Lunge, Pye]itis sinjstra.

2. 30jähriger lediger Bauer, Dementia praecox, war während seines elfmonatigen Anstaltsaufenthaltes ein sehr schwieriger Patient, zeitweise sehr aufgeregt mit kolossalem Geschrei, die Nahrung verweigernd, die Umgebung bolästigend; er musste daher sehr viel beschränkt werden. Stimmungswechsel oft sehr rasch. Einmal soll ein epileptiformer Anfall vorgekommen sein. Er versuchte sich wiederholt Selbstverletzungen beizubringen, so einmal die Augen mit den Fingern herauszustechen. Am 27. 1. 90 hatte er sich an einem Ofentürchen aufgehängt, wurdo aber noch rechtzeitig befreit. Manchmal arbeitete er wieder sehr fleissig in der Holzhütte. 25. 5. Pat. wusste sich in die Zelle ein Taschentuch mitzunehmen, mit dem er sich dann in den Maschen des 
Drahtgitters der Fensterbalken erhängte. Leicheneröffnungsergebnis: Strangulationsfurche. Chronisches Oedem der Meningen, Atrophia cerebri, Hydroceph. chron. int. Suffocatio ex strangulatione, Hämorrhagien im Brustfell, im retroösophagischen Zellgewebe, Nierenbecken und im Unterlappen der linken Lunge.

3. Hier handelt es sich um einen 39jährigen Schneidermeister, um einen sehr gewalttätigen, degenerierten, schwerer Trunksucht ergebenen Menschen, der mit dem Strafgesetz in Konflikt geriet wegen Verführung seiner eigenen Tochter aus erster Ehe zur Unzucht und wegen Uebertretung gegen die hörperliche Sicherheit. Pàt. hatte einen in den letzten Lebensjahren schwermütigen Vater, eine Schwester der Mutter war geistesschwach, ein Bruder war geistesgestört, ein anderer Bruder ein auffahrender, gewalttätiger Mensch. Am 21. 8.91 gab es nach reichlich genossenem Schnaps wieder eine schreckliche häusliche Szene zwischen ihm und seiner ebenfalls trunksüchtigen zweiten Frau, in deren Verlaufe es ausserordentlich wüst herging und wobei er schliesslich seinen zwei kleineren Kindern mit einem Rasiermesser den Hals abschnitt, sich selbst aber auch lebensgefährliche Schnitte am Hals beibrachte. - Die gerichtsärztliche Untersuchung wies seine Unzurechnungsfähigkeit nach und Pat. wurde am 20. 4. 92 unserer Anstalt übergeben. Hier beschäftigte er sich sehr fleissig mit Schnejderei, war aber in seinen Entlohnungsforderungen unverschämt. Gegenüber anderen Kranken war er ausserordentlich grob, spielte den Ueberlegenen. Gemütlich waren doch $\mathrm{ab}$ und zu Wehmutsanwandlungen bezüglich seiner Mordtaten zu bemerken. Mitunter schimpfte er wieder über allos in schonungslosester Weise. Am 7. 7.93 durchtrennte er sich mit einem Messer den Hals und eröfnete dabei die Trachea zwischen dem zweiten und dritten Trachealring. Nach der Durchtrennung steckte Pat. das Messer noch in den Sack und hängte sich mit einer Schnur aus Futterstoff gedreht auf. Es wurde sofort eine Kanüle eingeführt, die Blutung gestillt und vernäht, um 11 Uhr nachts trat jedoch der Tod ein. Obduktionsergebnis: Hamorrhagie in die Lunge infolge Aspiration von Blut nach der Durchtrennung der Schilddrüse und der Luftröhre. Anämie des Gehirns, Ependymitis granulosa. Trehirngewicht $1600 \mathrm{~g}$. Je ein linsengrosses zackiges Osteom in der Arachnoidea beider Stirnlappen.

4. Mit Dementia praecox behafteter Bauernknecht, 42 Jahre alt, war über 8 Jahre in der Anstalt. Er hatte quälende Sinnestäuschungen des Gesichts, Gehörs und Geruches, über den Teufel, er selbst sei der Antichrist, hatte hypochondrische Wahnideen, sprach in absonderlichen Ausdrücken. Dabei beherrschte ihn ein ganz ausserordentlicher Selbstmordtrieb. Wiederholt wurden derartige unternommene Versuche verhindert. Er machte dann die Bemerkung: Er sollte es halt doch einmal "derpacken". Ab und zu wurde er auch zu Gartenarbeiten unter strenger Aufsicht verwendet.

16. 4. 96. Es war Traktpflegerwechsel auf der Abteilung des Pat. Im Vorraum der Abteilung, wo Brunnen und Abort sich befindet, hielt sich vielfach ein ganz harmloser Mitkranker auf. Dieser öffnete an diesem Tage dem Pat. den Raum und liess es ganz ruhig zu, wie dieser sich mit einem Spüllappen erhängte.

Bei der Obduktion wurde gefunden: Leptomeningitis chron. productiva. Pachymeningitis ext. adhaesiva. Anaemia, Suffocatio ex strangulatione, Haemor- 
Die in den Jahren 1888 bis 1904 in Hall in Tirol Verstorbenen usw. 417

rhagia textus cellulosi retrooesophagei et retroaortici. Pleuritis chron. sinistra. Hypertrophia ventriculi sinistri cordis. Aorta auffallend eng $(6 \mathrm{~cm})$.

5. Ein schrullenhafter schizophrener:36 jähriger Bauernbursch machte sich wiederholt Selbstverletzungen. Am 12.2.97 brachte er sich frühmorgens im Bett tiefe Schnittwunden am Halse bei, die bis zum Kehlkopf vordrangen, und zwar mit einem im Abort zugeschliffenen Löffelstiel. Pat. brauchte zur Vollendung seiner Tat 2 Stunden. Verhielt sich ganz apathisch. Am 22. 5. sprang er mit dem Schädel an die Mauer im Abort; Impression der Schädeldecke in der Scheitelhöhe. Es stellte sich Pupillenstarre, rechtsseitige Facialislähmung und klonische Krämpfe der oberen Extremitäten ein. Am nächsten Tag exitus.

Kompression der rechten Grosshirnrinde durch extradurale ausgedehnte Blutung infolge Fissur des rechten Schläfenbeins, Stirnbeins und Keilbeinflügels mit Eröffnung der Art. meningea media dextra. Anämie des Gehirns. An der Innenfläche des Kehlkopfes eine über $1 \mathrm{~cm}$ lange zickzack-lineare Narbe. Bronchitis purulenta, Pneumonia lobularis utriusque et Oedema pulmonum.

6. Bei einem 42 jährigen Alkoholiker fehlt die Krankengeschichte. Im Sektionsprotokolle findet sich: Chron. Oedem der Meningen, Pachymening. externa adhaesiva und umschriebene interna haemorrhagica. Stauung im Gehirn, ebenso in Leber, Milz, Nieren, leichte exzentrische Hypertrophie des rechten Herzens bei Emphysem der Lunge. Ausgeprägte Strangulationsfurche am Hals.

7. Eine 45 Jahre alte, melancholische Taglöhnerin wurde trotz ärztlicher Mahnung - sie war sehr selbstgefährlich - vom Wartepersonal mit einer anderen Kranken allein im Garten gelassen, da Pat. durch ihren eintönigen Jammer auf der Abteilung grosse Störung verursachte. Um 2 Uhr nachmittags des 26. 8. 01 erhängte sie sich mit einem abgerissenen Schürzenteile am Gitterwerk einer Lichtöffnung und wurde tot angetroffen.

Bei Strangulation: Leichte exzentrische Hypertrophie, besonders des rechten Herzens, mässiges Oedem der Lunge, Hydropericardium und geringer Hydrothorax und Hydrops ascites. Verdichtung der Milz, zyanotische Induration der Nieren, Stauungserscheinungen in der Leber und besonders Staungskatarrh des Darmes. - Pachymeningitis externa, Oedem der Meningen und des Gehirns. Atrophie des Gehirns. Besonders in den Stirnteilen chronische Stauung und mässiges Oedem.

8. P. M., 30 jähriger Bauernsohn, Katatoniker, beging am 30. 8. 03 Selbstmord. In der Krankengeschichte findet sich keine abschliessende Bemerirung. Das Sektionsprotokoll führt an: Verblutung nach Eröffnung der linksseitigen Halsgefässe und Erstickung infolge Aspiration von Blut. Leichte äussere Pachymeningitis adhaes. Oedem der Meningen, passive Hyperämie des Gehirns, leichte Atrophie der Rinde, Erweiterung der Gefässe derselben. Persistenz der Thymus.

Von den 8 Selbstmordfällen kamen 5 auf die Dementia praecox.

X. Ohne nachweisbare Todesursache.

Ohne nachweisbare zu Tode führende Organerkrankung finden sich zwei Frauen; $\mathbf{0 , 5 5}$ pCt. 
T a

\begin{tabular}{|c|c|c|c|c|c|c|c|c|c|c|c|c|c|c|}
\hline Psychose & 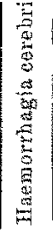 & 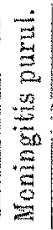 & 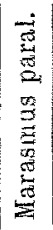 & 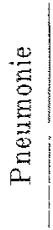 & 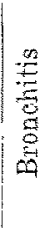 & 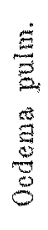 & 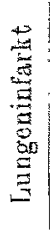 & : & 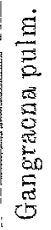 & $\begin{array}{l}\frac{.9}{3} \\
\frac{5}{5} \\
\frac{3}{2}\end{array}$ & 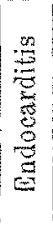 & 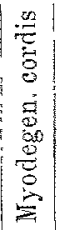 & 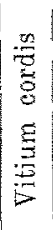 & 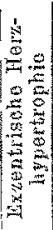 \\
\hline Delirien & & 8 & - & $\ldots$ & - & m & - & - & - & 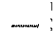 & - & - & - & - \\
\hline Alkoholismus. & 一 & - & $\ldots$ & $\ldots$ & - & 1 & - & - & 一 & - & - & ــ & - & - \\
\hline Dementia praecos. . . & - & 1 & - & 15 & 1 & - & - & $\ldots$ & 3 & 1 & - & 4 & 4 & - \\
\hline Dementia paranoides. & 1 & - & - & 4 & 1 & - & - & 一 & 1 & - & - & 3 & 一 & - \\
\hline Dementia paralytica. . & $-m$ & 1 & 2 & 52 & 6 & 13 & 2 & 1 & 3 & $-m$ & - & 3 & 一 & - \\
\hline Lues cerebri . . . . . & - & - & - & - & - & - & - & - & $\longrightarrow$ & $-\cdots$ & - & 一 & - & $-m$ \\
\hline Arteriosklerotische Ge- & & & & & & & & & & & & & & \\
\hline hirnerkrankung $\ldots$ & - & - & - & 1) & - & - & - & - & - & $-\cdots$ & - & - & - & $\longrightarrow$ \\
\hline Dementia apoplectiea . & $\longrightarrow$ & - & - & 1 & - & - & - & - & - & - & - & - & - & - \\
\hline Prexsenile Demenz .... & - & - & - & 1 & - & - & - & 一 & - & - & - & - & - & - \\
\hline Dementio senilis. . . . & 2 & 1 & - & 18 & 1 & 2 & - & - & - & 1 & - & 2 & $\cdots$ & $一$ \\
\hline Mau.-depress. Irresein . & 1 & - & - & 201 & 4 & 2 & 1 & - & 1 & 1 & 1 & 1 & - & $\rightarrow$ \\
\hline Paragoia ....... & - & - & - & -1 & 一 & - & - & 一 & $\longrightarrow$ & $m$ & - & 1 & - & - \\
\hline Epileptisches Irresein. & $-m$ & $-\cdots$ & - & 4 & 3 & 1 & - & - & 2 & - & - & 一 & 一 & $-m$ \\
\hline Hysterie ...... & - & - & - & -1 & - & 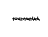 & - & - & - & - & - & - & - & $\rightarrow$ \\
\hline Neurasthenie...... & 一 & - & - & - & - & $\ldots$ & - & - & 一 & - & - & 一 & $一$ & - \\
\hline Degeneratives Irresein . . & 一 & - & - & - & - & - & - & - & - & - & - & - & - & - \\
\hline Imbenilität $\ldots \ldots$ & - & - & - & 2 & - & - & - & - & 一 & - & - & - & - & - \\
\hline rdiotie ........... & $\ldots$ & - & 一 & 2 & - & - & - & - & $\ldots$ & 1 & - & - & - & - \\
\hline Tumor cerebri.... & w & - & - & 1 & - & - & - & - & 一 & 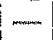 & - & - & - & - \\
\hline Irresein bei Pellagra. & - & - & - & 一 & - & $-m$ & - & - & - & 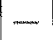 & - & 1 & - & - \\
\hline Unbestimmte Diagnose & - & - & - & 3 & - & $=$ & - & -- & 1 & - & - & 1 & - & 1 \\
\hline$\theta 2$ & 4 & 5 & 2 & 124 & 16 & 19 & & & 11 & & 1 & 16 & 7 & \\
\hline
\end{tabular}

Die Krankengeschichte der ersteren, einer 17 Jahre alten hebephrenen. Patientin ist nicht auffindbar. Dieselbe sank beim Essen plätzlich tot um. Im Verwaltungsprotokoll heisst es „Schlagfluss“. Die Sektion ergab jedoch: Aussergewöhnliche Enge der Aorta und der arteriellen Ostien des Herzens. Induration und Stauung der Nieren. Hydroperikard, Brouchitis catarrh. Leichter MagenDarmkatarrh. Cystitis catamh. Menstruelle Elchymosen des Utorus, dessen Sohloimhaut aufgelookert ist. Anaemia generalis. Pachymeningitis externa adhaesiva, leichtes Oedem dex Meningen.

Die zweite, A. N., 35 Jahre alt, war eine ausserordentlich reizbare, sehr aufgeregte, wiederholt ron schweren Anfällen heimgesuchte Epileptikerin. Sie war von Haus aus sehwachsinnig, zu Exzessen in baccho et vonere geneigt. Das Leiden bestand angeblich seit 10 Jahren. Am 18.9 .03 um 4 Whr frîh hörte die Pllegerin sie nooh mit anderen Patienten sprechen, auch firanke berichteten, dass die Verstorbene um 4 Uhr noch munter gewesen. Dann habe sie sich nach der Seite gekehrt und vollkommen ruhig verhalten. Als um à Uhr aufgestanden wurde, erhob sich Pat. nicht und man fand, dass sie tot war. Die vorgenommene Leichenöffnung stellte fest: Kleinste Blutumgen in dex Pleura und 
belle XIII.

\begin{tabular}{|c|c|c|c|c|c|c|c|c|c|c|c|c|c|c|c|c|c|c|c|}
\hline 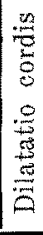 & 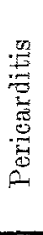 & 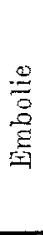 & 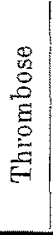 & 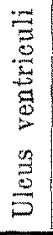 & 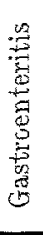 & $\stackrel{3}{3}$ & 要 & $\frac{\sqrt[\infty]{3}}{\stackrel{5}{5}}$ & 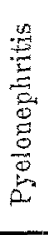 & 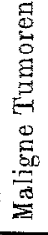 & 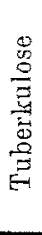 & 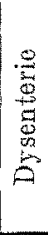 & $\frac{\frac{n}{3}}{\frac{m}{n}}$ & م & 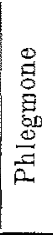 & 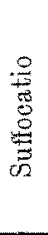 & 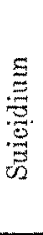 & 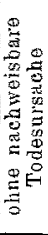 & 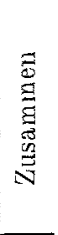 \\
\hline$\rightarrow$ & - & - & - & $\longrightarrow$ & - & - & - & - & - & - & 1 & - & - & - & - & - & - & & \\
\hline- & - & $\ldots$ & - & - & - & - & - & 1 & - & - & - & - & - & - & - & - & 1 & $\pi$ & \\
\hline- & 2 & - & - & 1 & 1 & - & 1 & 2 & $\sim$ & 5 & 52 & 1 & 5 & $\ldots$ & - & - & 5 & 1 & 105 \\
\hline- & 1 & - & - & - & - & - & 1 & - & - & 2 & 3 & - & - & - & - & - & $\cdots$ & - & 1 \\
\hline$\ldots$ & - & - & 1 & - & $\rightarrow$ & - & 3 & - & 1 & - & 10 & - & - & 2 & - & 5 & - & 一 & 105 \\
\hline & - & - & - & - & - & - & - & 1 & 一 & - & - & $1-$ & - & - & - & - & - & - & 1 \\
\hline- & - & - & - & - & - & - & - & - & $\cdots$ & - & - & - & - & - & $\ldots$ & - & - & - & 1 \\
\hline & - & 1 & - & - & - & - & - & - & 一 & 1 & 一 & - & - & - & - & - & - & 一 & $\stackrel{a}{a}$ \\
\hline & $\sim$ & - & - & - & - & - & - & - & - & $\cdots$ & - & - & - & - & - & - & - & 一 & 1 \\
\hline 1 & - & - & - & - & - & - & 2 & - & - & - & 4 & 1 & - & - & - & - & - & - & 35 \\
\hline- & - & 2 & - & - & - & 1 & 1 & - & 一 & 6 & 12 & 1 & - & 1 & - & 1 & 1 & - & 58 \\
\hline- & - & 一 & - & - & - & - & - & - & - & 一 & - & - & - & - & - & - & - & - & 1 \\
\hline- & - & - & 一 & - & - & - & - & - & - & - & 3 & 1 & - & - & 1 & 1 & - & 1 & 17 \\
\hline$\cdots$ & - & - & - & - & - & - & - & $-m$ & - & - & 1 & - & 1 & - & - & - & $-\cdots$ & - & 2 \\
\hline- & - & - & - & - & - & - & - & - & - & 1 & - & - & - & - & - & - & - & - & 1 \\
\hline$\cdots$ & - & - & - & - & - & - & - & - & 一 & $\cdots$ & 1 & - & - & - & $\longrightarrow$ & - & 1 & - & 2 \\
\hline- & - & - & - & - & - & - & - & - & - & - & 6 & - & - & - & - & - & - & - & 8 \\
\hline- & - & - & - & - & - & - & - & - & - & - & 1 & - & - & - & 一 & - & - & - & 4 \\
\hline- & - & 一 & - & - & - & 一 & - & - & - & - & - & - & - & - & - & - & - & - & 1 \\
\hline- & - & 一 & - & - & - & - & - & w & - & - & - & - & - & - & $\rightarrow$ & - & - & - & 1 \\
\hline- & 1 & - & - & 1 & - & - & - & - & -- & - & 5 & 1 & - & - & - & - & - & - & 14 \\
\hline 1 & 4 & 3 & 1 & 2 & 1 & & & & 1 & 15 & 99 & & 0 & 3 & 1 & & & & 0 \\
\hline
\end{tabular}

im Perikard. Passive Hyperämie der Lungen und der grossen Bauchdrüsen. Pachymeningitis externa adhaesiva chron., Hyperämie der Meningen.

Tabelle XIII veranschaulicht übersichtlich das Verhältnis zwischen Psychosen and Todesursachen.

Und so bin ich nun am Schlusse meiner Arbeit, anf deren Ergebnis ich wohl nicht mehr zuruckzukommen brauche. - Ich sah meine Aufgabe auch darin gelöst, dass ich das vorhandene Material nach diesem Gesichtspunkte durchforscht habe. Ein Teil des Wertes der Arbeit dürte darin liegen, dass unsere Kranken grossenteils aus der vielfach noch urwüchsigen Landbevölkerung Tirols stammten, über welche wenjg diesbezügliches Statistisches veröfenticht wurde. Nur eine Zahl erscheint auffallend: $23,88 \mathrm{pCt}$. der Verstorbenen waren Paralytiker! Doch muss ich gleich beifügen, dass das Gros dieser Kranken eben aus den grösseren Städten und deren nächster Umgehung, speziell Insbruck, war, wie schon seinerzeit unser Oberarzt, Dr. Georg Eisath, nachgewiesen hat, und worauf ich vielleicht anch noch einmal zurickkommen werde. 